\title{
INTERIM PERFORMANCE FEEDBACK IN MULTISTAGE TOURNAMENTS: THE OPTIMALITY OF PARTIAL DISCLOSURE*
}

\author{
MARIA GOLTSMAN ${ }^{\dagger}$ AND ARIJIT MUKHERJEE ${ }^{\ddagger}$
}

\begin{abstract}
Workers competing in a tournament for a given prize, say a promotion, often perform sequentially in multiple stages. When the firm is privately informed about the workers' performance, it can sharpen incentives by strategically disclosing the intermediate results. But the policies that enhance final-stage effort may dampen incentives at the intermediate stage. In our model, the optimal disclosure policy has a simple form: disclose only if all workers perform poorly. This result offers a novel justification for partial disclosure in performance feedback. Also, it is in contrast with the existing literature that focuses on the extreme policies of 'full disclosure' and 'no disclosure.'
\end{abstract}

\section{INTRODUCTION}

While tournaments have received significant attention in personnel economics (Green and Stokey, 1983; Lazear and Rosen, 1981; Nalebuff and Stiglitz, 1983), the existing literature has mostly focused on static tournaments. In these tournaments, the winner is determined by how well the participants perform a single task. But tournaments are often dynamic in nature (Meyer 1991, 1992; Rosen, 1986). Moreover, in many dynamic tournaments, the firm (or the tournament organizer) is more informed about the workers' (or the contestants') progress compared to what the workers can infer by themselves.

Consider the example of a promotion tournament. In order to be eligible for a promotion, a worker usually spends a certain duration of time in his current position, and his promotion depends on how well he has performed relative to his peers in all the tasks that he has been responsible for. But in the absence of any objective performance measure, the supervisor of the worker may be better informed about the worker's (and his peers') performance compared to what the worker might learn by himself. In fact, when the performance measure is subjective, promotion tournament might be the only credible incentive mechanism that the firm can offer, because the subjective nature of the performance measure renders all pay-for-performance contracts (both explicit and relational) infeasible (Carmichael, 1983b; Malcomson, 1984).

In a multistage tournament, the information advantage of the firm allows it to strategically disclose information to the workers to affect their work incentives. The strategic disclosure of information is particularly relevant as an incentive device when the firm has little flexibility in choosing the rewards of the tournament. Such is the case, for example, when the workers'

Date: April 15, 2010.

*We would like to thank Chris Ahlin, Braz Camargo, Carl Davidson, Florian Ederer, John Hollenbeck, Giuseppe Lopomo, Zsolt Macskasi, Bentley MacLeod, Gregory Pavlov, Gary Solon, Michael Waldman, Jan Zábojník, two annonymous referees, the participants at the Tournaments, Contests and Relative Performance Evaluation conference, North Carolina State University, Raleigh, and the 2009 NASM of the Econometric Society, Boston University, Boston, for their helpful comments. An earlier version of this article was circulated under the title "Information disclosure in multistage tournaments." All errors that remain are ours.

${ }^{\dagger}$ Department of Economics, University of Western Ontario, 1151 Richmond St N, London, ON N6A 5C2, Canada. Email: mgoltsma@uwo.ca. URL: http://www.ssc.uwo.ca/economics/faculty/goltsman/

${ }^{\ddagger}$ Department of Economics, Michigan State University, 110 Marshall Adams Hall, East Lansing, MI 48823, USA. E-mail: arijit@msu.edu. URL: http://www.amukherjee.net. 
post-promotion wages are market determined (Waldman, 1984). When the firm is privately informed about how well each worker has performed in the intermediate tasks, it can choose how much feedback to give to the workers. In this context, we ask the following question: What is the optimal disclosure policy when the firm attempts to maximize the total effort exerted by its workers?

Indeed, most firms today adopt some form of performance appraisal and feedback policy to motivate their employees. ${ }^{1}$ However, how much information the supervisors should disclose to their subordinates is a highly debated issue. Some scholars have argued that a greater openness in the feedback system facilitates trust in the reward system (Hammer, 1975; Lawler, 1987). Others have argued that too much of disclosure may be counterproductive, because it may damage self-esteem and thereby demotivate the employees (Beer, 1990).

This paper makes two contributions to a nascent but growing literature on optimal interim feedback (Lizzeri et al., 2002; Yildirim, 2005; Fuchs, 2007; Gershkov and Perry, 2009; Hansen, 2009; Aoyagi, 2010; Dubey and Geanakoplos, 2010; Ederer, 2010). First, we highlight a new trade-off associated with the interim performance disclosure in a tournament: a disclosure policy that enhances post-disclosure work incentives may dampen work incentives in the predisclosure stage. While the existing literature has recognized that the firm's disclosure policy can affect both the pre- and the post-disclosure effort levels in a tournament (e.g., Yildirim, 2005; Aoyagi, 2010; Ederer, 2010), it has not fully explored the potential trade-off that may arise between the two. ${ }^{2}$

Second, we show that, in the face of the aforementioned trade-off, partial disclosure can be strictly optimal. This finding is in contrast with the existing literature. The existing models that study information disclosure in tournaments either a priori restrict attention to the 'extreme' policies of full disclosure and no disclosure (Yildirim, 2005; Ederer, 2010) or show that one of the extreme policies is always optimal, depending on the curvature of the workers' cost function (Aoyagi, 2010). Our results indicate that limiting attention only to the extreme policies may imply a loss of generality, and that the optimal policy can be more nuanced than a simple choice between full and no disclosure.

Partial disclosure in the form of "grade compression" is widespread in performance appraisal: while rating their employees' performances, supervisors often give a very coarse rating (Beer, 1990; Murphy, 1990; MacLeod, 2003). A number of recent articles provide explanations for this phenomenon in contexts that are quite different from ours. For example, Dubey and Geanakoplos (2010) consider a one-stage game where each participant's outcome is a noisy function of his effort, and participants care about how their performance compares to that of others. They show that coarse feedback that pools agents with similar performances (e.g., letter grades as opposed to exact numerical scores in an examination) may generate better incentives than full disclosure. Fuchs (2007) considers a dynamic principalagent model with subjective performance evaluation. He shows that it may be optimal for the firm not to offer any feedback unless the worker's performance falls below a threshold, at

\footnotetext{
${ }^{1}$ A commonly observed feedback system in many organizations is the so-called " $360^{0}$ feedback" system. In this system the supervisor gathers information about his employee not only based on his own observation, but also by seeking performance appraisals about the worker from his peers. See Peiperl (2001) for a discussion on the pros and cons of such a system. Also see Murphy $(1990,1992)$ for a case study on a more traditional feedback system where each manager offers feedback to her employee solely based on her own observation about the employee's performance.

${ }^{2}$ Gershkov and Perry (2009) highlight a similar tradeoff in a tournament model where the principal decides how much weight to put on the midterm review, but assumes an exogenously fixed disclosure policy. Lizzeri et al. (2002) compare full disclosure to no disclosure in a principal-agent model with interim performance evaluation and find that, for certain wage schemes, full disclosure induces higher pre-disclosure effort but lower post-disclosure effort. Our article is perhaps the first one to study this tradeoff in the context of a tournament where the principal chooses a disclosure policy.
} 
which point the worker is fired. Hansen (2009) proves the optimality of partial disclosure in a principal-agent model with career concerns, where interim feedback provides the agent with information about his own ability. Our result suggests that partial disclosure can also arise as part of an optimal incentive structure in a dynamic tournament even when it is common knowledge that the participants have the same abilities.

We consider a simple model of promotion tournament where two ex ante identical workers compete in two stages, intermediate and final. At each stage, each worker can either succeed or fail. After the intermediate stage, the firm (but not the workers) observes the results and publicly discloses some information about them. After observing the firm's disclosure, the workers update their beliefs about the intermediate outcome and choose their final-stage effort levels. The worker who gets the highest number of successes over the two stages is promoted. At the beginning of the game, the firm commits to a disclosure policy, which maps the interim performances of the workers into a set of signals that the firm can publicly disclose. The post-promotion wages are exogenous to the firm and the only channel through which the firm can influence the effort incentives is by strategic disclosure of the intermediate performance. $^{3}$

The key result of our paper is that the optimal disclosure policy takes a simple form: the firm discloses information (without any noise) only if both workers fail at the intermediate stage, and does not disclose any information following any other intermediate outcome. The intuition behind this result is the following. While choosing the optimal disclosure policy, the firm faces a trade-off between final-stage and intermediate-stage effort: a disclosure policy that enhances the final-stage effort may dampen the intermediate-stage effort incentives. When the workers learn that the race is close, the final-stage efforts are high, because by exerting additional effort a worker can significantly affect his chances of getting promoted. But effort is costly, and consequently a closely competed race lowers the participants' expected payoffs. So, if the disclosure policy informs the workers whenever the race is close, at the intermediate stage the workers may also have a countervailing incentive to reduce their effort in order to avoid a close race at the final stage. The disclosure policy "disclose only if both fail" resolves this trade-off optimally. On the one hand, disclosing the information that both workers have failed at the intermediate stage (and thus are in the same position) stimulates competition at the final stage. On the other hand, both workers would not want to find themselves in the position where they compete intensively at the final stage. So they have incentives to exert effort at the intermediate stage in order to decrease the probability of the outcome where they both fail.

To establish this result, we first consider the disclosure policies that are symmetric (immune to any permutation of the workers' names) and deterministic (the feedback depends deterministically on the intermediate results). This class of disclosure policies is not only more realistic, it is also analytically tractable. We then analyze the optimal disclosure policy in the class of all feasible disclosure policies. This problem is analytically intractable because of the potentially large set of possible signals. However, we show that one can bound the required number of signals, which allows for numerical tractability. The numerical optimization results indicate that the partial disclosure policy discussed above remains optimal even if the firm can choose any general policy.

This paper is organized as follows. The next section presents the model. Section 3 characterizes the optimal effort choice given any disclosure policy. Section 4 analyzes the optimal disclosure policy and Section 5 discusses the robustness of the main findings to some alternative modeling assumptions. The final section concludes.

\footnotetext{
${ }^{3}$ In this sense, our article complements the earlier literature on dynamic tournaments (Rosen, 1986; Meyer, 1991, 1992) that assumes exogenous information structure and studies the optimal reward structure.
} 


\section{The ModeL}

We present a model of multistage promotion tournament that is described below in terms of the following key components: players, technology, incentives and payoffs.

Players: A firm (principal) $F$ hires two workers (agents) $A$ and $B$. Both workers are staffed in a given job.

TECHNOlOGY: The job must be performed in two stages, intermediate and final. ${ }^{4}$ The production technologies in each of the two stages of the job are identical. In each of the two stages, both workers simultaneously choose how much effort to exert. At the intermediate stage, worker $i(i \in\{A, B\})$ exerts effort $e_{i} \in[0,1]$ at the cost $c\left(e_{i}\right)=e_{i}^{2} / 2$. The outcome $y_{i}$ of the worker's effort can either be a success $(s)$ or a failure $(f)$ and depends on the exerted effort, where

$$
\operatorname{Pr}\left(y_{i}=s\right)=e_{i} .
$$

We assume that the performance of a worker in both stages is private information to the firm. The workers do not observe the outcome of the intermediate stage (own outcome as well as the outcome of the other worker), but the firm observes the outcome for both workers perfectly. That is, $F$ observes an element of the set $\mathcal{Y}=\{(s, s),(s, f),(f, s),(f, f)\}$, where, for example, $(s, f)$ corresponds to the outcome where worker $A$ succeeds and worker $B$ fails at the intermediate stage. This would be the case, for example, where there are no objective measures for the workers' performance, and the firm uses a subjective measure to assess the workers' output. The firm, however, can disclose some (potentially noisy) information to the workers at the beginning of the final stage. We will elaborate on the disclosure policy shortly.

At the beginning of the final stage, both workers observe the disclosed information. Worker $i(i \in\{A, B\})$ exerts effort $E_{i} \in[0,1]$ at the cost $c\left(E_{i}\right)=E_{i}^{2} / 2$. Similar to the intermediate stage, a worker produces the final stage outcome $Y_{i} \in\{s, f\}$, where

$$
\operatorname{Pr}\left(Y_{i}=s\right)=E_{i}
$$

We also assume that at both stages the levels of effort are unobservable. Note that the performances of the two workers at both stages are statistically independent of each other.

InCEntives AND Disclosure POLICY: The firm would like to induce the workers to exert effort in both stages of the job. But pay-per-performance contracts are not feasible in this environment since the worker's performance is private information to the firm: the firm always has an incentive to report low performance in order to save on the wage payment. However, the firm can credibly offer a promotion tournament, because the promotion decision is publicly observed. ${ }^{5}$ Namely, the firm can commit to promote one of the two workers - the

\footnotetext{
${ }^{4}$ The technology specification extends the model of Lizzeri et al. (2002) to a tournament setting.

${ }^{5}$ This idea is due to Carmichael $(1983 \mathrm{a}, \mathrm{b})$ and Malcomson $(1984,1986)$ : when the workers' performance is firm's private information, a tournament contract can serve as a credible incentive device, whereas pay-perperformance contracts lack credibility. However, it is important to note that in a repeated game, promotion tournament need not be the only incentive mechanism available to the firm. In a repeated game setting, the firm can offer relational contracts promising bonus payments to reward good performance. Such contracts can be sustained as an equilibrium of the repeated game and provide work incentives, although they must imply surplus destruction even on the equilibrium path (see MacLeod, 2003, and Fuchs, 2007). In our model, we do not consider repeated interactions between the firm and the workers, and hence, rule out relational contracts in order to focus on the incentive role of information feedback.
} 
one with the highest number of successes over the two stages - to a new job at the end of the game.

In order to focus on the incentive implications of the information feedback, we assume that the rewards of the winner and the loser of the promotion tournament are exogenously fixed. ${ }^{6}$ The worker who "wins" the tournament (by scoring the most number of successes over the two stages) gets promoted and earns an exogenously specified reward (or the wage offered in the new job) of 1 , while the other worker (the one with the lower number of successes among the two) "loses" the tournament and earns 0. We assume that in case of a tie, each worker is equally likely to be promoted. ${ }^{7}$ In addition, the firm can offer lump-sum wages $W_{A}$ and $W_{B}$ to workers $A$ and $B$ at the beginning of the game. We assume that the workers are liquidity constrained, i.e., $W_{i} \geq 0$, for $i=A, B$.

Note that in this environment, even if the firm cannot manipulate the tournament's rewards, the firm can sharpen incentives by strategically disclosing the information about the intermediate-stage outcome to the workers. The information about the intermediate outcome can affect how much effort the workers exert at the final stage, and therefore, their equilibrium behavior at the intermediate stage as well. The information disclosure mechanism is defined as follows.

At the beginning of the game, $F$ commits to a disclosure policy $(Z, \zeta)$ that maps the intermediate outcome of both workers, $\left(y_{A}, y_{B}\right)$, into a set of public signals $Z$ according to the function $\zeta: \mathcal{Y} \rightarrow \Delta Z$. In other words, at the end of the intermediate stage, depending on the output pair $\left(y_{A}, y_{B}\right)$, a public signal $z \in Z$ is realized that is drawn from the probability function $\zeta\left(z \mid y_{A}, y_{B}\right)$. In what follows, we will denote $\zeta\left(z \mid y_{A}, y_{B}\right)$ by $\zeta_{y_{A} y_{B}}(z)$.

Note that the disclosure policy defined above is completely general in its form. As special cases, it includes no disclosure (the firm sends the same signal regardless of the realized outcome), full disclosure (there is a one-to-one correspondence between intermediate outcomes and the signals that are disclosed following these outcomes), and all other deterministic disclosure policies (such that the probability distributions $\zeta_{y_{A} y_{B}}(z)$ are degenerate). Moreover, it also allows the firm to introduce some noise to its report by randomizing over multiple signals using a pre-specified probability distribution.

PAYOFFS: We assume that both the workers and the firm are risk neutral. The expected payoff of worker $i$ is

$$
\begin{aligned}
& U_{i}\left(W_{i}, \mathbf{e}, \mathbf{E}\right)= \\
& W_{i}+\operatorname{Pr}(i \text { wins } \mid \mathbf{e}, \mathbf{E})+\frac{1}{2} \operatorname{Pr}(\text { tie } \mid \mathbf{e}, \mathbf{E})-\frac{1}{2} e_{i}^{2}-\sum_{\left(y_{A}, y_{B}\right) \in \mathcal{Y}} \operatorname{Pr}\left(y_{A}, y_{B}\right) \int_{Z} \frac{1}{2} E_{i}(z)^{2} d \zeta_{y_{A} y_{B}},
\end{aligned}
$$

where $\mathbf{e}=\left(e_{A} ; e_{B}\right)$ and $\mathbf{E}=\left\{E_{A}(z), E_{B}(z)\right\}$. That is, the payoff of a worker is equal to the expected reward he earns at the end of the tournament net of his expected cost of effort in both stages. We assume that the outside option of a worker is 0 .

The firm's payoff, $\Pi$, is the expected effort exerted by the workers $A$ and $B$ in the two stages of production net of the wage payments ${ }^{8}$, i.e.

\footnotetext{
${ }^{6}$ In the Appendix, we present a slightly modified version of this model to motivate our assumption of the inflexible tournament reward structure. The key idea is that the reward structure can be perceived as market determined (and hence, exogenous to the firm), the market inferring the workers' productivity from the promotion decision (a la Waldman, 1984). Also see Section 5 for further discussion on the robustness of our findings to alternative reward structures.

${ }^{7}$ We consider alternative tie-breaking rules in Section 5.

${ }^{8}$ One way to interpret this formulation of the firm's payoff is to assume that the firm's profit is deterministically governed by the level of effort put in by the two workers, and the outcome of each stage $\{s, f\}$ is a signal of the workers' effort and does not affect the firm's bottom line by itself. Alternatively, one can assume
} 


$$
\Pi=e_{A}+e_{B}+\sum_{\left(y_{A}, y_{B}\right) \in \mathcal{Y}} \operatorname{Pr}\left(y_{A}, y_{B}\right) \int_{Z}\left(E_{A}(z)+E_{B}(z)\right) d \zeta_{y_{A} y_{B}}-W_{A}-W_{B} .
$$

Time Line. The timing in the game is as follows.

- Beginning of Period 1. F publicly announces wages $W_{A}, W_{B}$ and disclosure policy $(Z, \zeta)$. Workers decide whether to accept the contract.

- Period 1.1. Each worker $i(i=A, B)$ chooses the intermediate-stage effort $\left(e_{i}\right)$. The effort choice is simultaneous.

- Period 1.2. Intermediate-stage outcome $\left(y_{A}, y_{B}\right)$ is realized. $F$ observes $\left(y_{A}, y_{B}\right)$ and discloses a public signal $z$ according to the disclosure policy.

- Period 1.3. Each worker $i(i=A, B)$ observes $z$ and chooses the final-stage effort $\left(E_{i}\right)$. The effort choice is simultaneous.

- End of Period 1. Final-stage outcome $\left(Y_{A}, Y_{B}\right)$ is realized. $F$ aggregates the outcome for each worker over the two stages and the winner of the promotion tournament is announced. Wages $\left(W_{i}\right)$ and the tournament rewards are paid and the game ends.

Strategies And EQuilibrium COnCEPT: The strategy of $F$ is to choose wages $\left(W_{A}, W_{B}\right)$ and a disclosure policy $(Z, \zeta)$ at the beginning of the game. A worker's strategy has three components: (i) acceptance or rejection of the firm's contract, (ii) the choice of intermediatestage effort level, $e_{i}$, and (iii) the choice of final-stage effort level, $E_{i}(z)$, given the realized signal. We use the perfect Bayesian equilibrium (PBE) as the solution concept.

\section{Optimal EFFORT}

In order to derive the optimal disclosure policy, the first step is to characterize the optimal effort levels exerted by the workers for a given disclosure policy. This section discusses such characterization.

3.1. Best-response effort choices. Because a disclosure policy affects both pre- and postdisclosure effort incentives of the workers, we solve for the optimal effort for a given disclosure policy through backward induction. Fix a disclosure policy $(Z, \zeta)$. Given a disclosed signal $z \in Z$, worker $i$ 's choice of the final-stage effort, $E_{i}^{*}(z)$, maximizes his probability of winning the tournament conditional on $z$ net of the cost of final-stage effort. That is,

$$
E_{i}^{*}(z)=\arg \max _{\hat{E}_{i} \in[0,1]} \operatorname{Pr}\left(i \text { wins } \mid \hat{E}_{i}, e_{i}, E_{j}^{*}, z\right)+\frac{1}{2} \operatorname{Pr}\left(\text { tie } \mid \hat{E}_{i}, e_{i}, E_{j}^{*}, z\right)-\frac{1}{2} \hat{E}_{i}^{2}
$$

The first-order conditions with respect to $E_{A}(z)$ and $E_{B}(z)$ can be written as: ${ }^{9}$

$$
E_{A}(z)=E_{A}\left(E_{B}, e_{A}, z\right) \text { and } E_{B}(z)=E_{B}\left(E_{A}, e_{B}, z\right)
$$

Denote the solution to (2) by $\mathbf{E}^{*}(z)=\left\{E_{A}^{*}(z), E_{B}^{*}(z)\right\}$. The expression for $\mathbf{E}^{*}(z)$ is given by equations (Final) in the Appendix.

The intermediate-stage effort choice of a worker, $e_{i}$, maximizes his probability of winning the tournament, given the disclosure policy, the strategy of the opponent and the final-stage effort choices of the two workers, $\mathbf{E}^{*}$ :

\footnotetext{
that the firm earns fixed payoffs from each success and failure by the two workers over the two periods and maximizes its expected payoff.

${ }^{9}$ The second-order conditions hold for both agents, because $\operatorname{Pr}(i$ wins $\mid z)$ is linear in $E_{i}(z)$ and the cost of effort is convex.
} 
(3)

$e_{i}^{*}=\arg \max _{\hat{e}_{i} \in[0,1]} \operatorname{Pr}\left(i\right.$ wins $\left.\mid \mathbf{E}^{*}, \hat{e}_{i}, e_{j}^{*}\right)+\frac{1}{2} \operatorname{Pr}\left(\operatorname{tie} \mid \mathbf{E}^{*}, \hat{e}_{i}, e_{j}^{*}\right)-\frac{1}{2} \hat{e}_{i}^{2}-\mathbb{E}_{z}\left(\frac{1}{2} E_{i}^{*}(z)^{2} \mid \mathbf{E}^{*}, \hat{e}_{i}, e_{j}^{*}\right)$.

The optimal intermediate-stage effort for both workers must satisfy the first-order conditions associated with the above maximization problem. Because the maximand in equation (3) depends on the final effort choice $\mathbf{E}^{*}(z)$, the first-order conditions with respect to $e_{A}$ and $e_{B}$ can be written as:

$$
e_{A}=e_{A}\left(e_{B} ; \mathbf{E}^{*}\right) \text { and } e_{B}=e_{B}\left(e_{A} ; \mathbf{E}^{*}\right)
$$

Denote the solution to (4) by $\mathbf{e}^{*}=\left\{e_{A}^{*}, e_{B}^{*}\right\}$. The complete expression for (4) is given in the Appendix (equations (Int)).

The following lemma shows that for any given disclosure policy, there always exists an equilibrium of the tournament subgame where the effort levels are characterized by the firstorder conditions (2) and (4).

Lemma 1. Given any disclosure policy $(Z, \zeta)$, there exists a PBE of the subgame played by the two workers that is induced by $(Z, \zeta)$, where the equilibrium levels of effort are equal to $\left\{\mathbf{e}^{*}, \mathbf{E}^{*}(z)\right\}$. Moreover, in equilibrium, the constraints $0 \leq e_{i} \leq 1$ and $0 \leq E_{i}(z) \leq 1$ do not bind for any $i \in\{A, B\}$ and $z \in Z$.

In the next section, we solve for the optimal disclosure policy of the firm.

\section{Optimal Disclosure Policy}

The firm's problem is to choose the wages $W_{A}, W_{B}$ and a disclosure policy $(Z, \zeta)$ that maximize the expected profit over the two stages subject to the following constraints: (i) incentive compatibility $(I C)$ : a disclosure policy induces an effort profile $\left\{\mathbf{e}^{*}, \mathbf{E}^{*}(z)\right\}$ that is a PBE of the game played by the two workers; (ii) individual rationality $(I R)$ : the expected payoff of a worker from accepting the firm contract is no less than his outside option, 0; (iii) liquidity constraint $(L C): W_{i} \geq 0$. The firm's problem is:

$$
\max _{W_{A}, W_{B},(Z, \zeta)} \quad \Pi
$$

s.t. $\left\{\mathbf{e}^{*}, \mathbf{E}^{*}(z)\right\}$ is a PBE of the game induced by $(Z, \zeta)$ where $\zeta$ is a probability function on $Z \forall\left(y_{A}, y_{B}\right) \in \mathcal{Y}$,

$$
\begin{gathered}
U_{i}\left(W_{i}, e_{i}^{*}, E_{i}^{*}(\cdot)\right) \geq 0 \text { for } i \in\{A, B\}, \\
W_{i} \geq 0 \text { for } i \in\{A, B\} .
\end{gathered}
$$

The following lemma simplifies the firm's problem by showing that the $(I R)$ constraint is always satisfied.

Lemma 2. Given any $W_{i} \geq 0$ and an arbitrary disclosure policy $(Z, \zeta)$, the payoff to a worker at the PBE effort levels $\left\{\mathbf{e}^{*}, \mathbf{E}^{*}(z)\right\}$ induced by $(Z, \zeta)$ is always non-negative. 
The argument behind this lemma is straightforward, and we omit the formal proof. The promotion tournament offers a non-negative reward to all participants. Hence, even if $W_{i}=0$, the worker can always guarantee a payoff of 0 by accepting the contract and not exerting any effort. So, as a part of a PBE, $\left\{\mathbf{e}^{*}, \mathbf{E}^{*}(z)\right\}$ must ensure a non-negative payoff to the workers.

This lemma has two implications: (i) If an effort profile $\left\{\mathbf{e}^{*}, \mathbf{E}^{*}(z)\right\}$ is a PBE of the game played by the two workers, it must also satisfy the $(I R)$ constraints for both workers even when $W_{A}=W_{B}=0$. (ii) The $(L C)$ constraint always binds, because the worker will always accept the offer even when $W_{i}=0$.

Given these two observations, the firm's problem can be simplified as follows:

$$
\mathcal{P}\left\{\begin{array}{c}
\max _{(Z, \zeta)} \Pi=e_{A}^{*}+e_{B}^{*}+\sum_{\left(y_{A}, y_{B}\right) \in \mathcal{Y}} \operatorname{Pr}\left(y_{A}, y_{B}\right) \int\left(E_{A}^{*}(z)+E_{B}^{*}(z)\right) d \zeta_{y_{A} y_{B}}(z) \\
\text { s.t. }(I C) .
\end{array}\right.
$$

The key technical challenge in solving the program $\mathcal{P}$ is that the set of feasible disclosure policies $(Z, \zeta)$ is large, because $Z$ can be an arbitrarily (potentially infinitely) large set of signals. ${ }^{10}$

We proceed in two steps. First, we restrict attention to a special class of disclosure policies, namely, symmetric and deterministic policies. Intuitively, a symmetric and deterministic disclosure policy is one that is invariant to permutations of the workers' identities, and maps each intermediate stage outcome into a unique signal. Under these policies, the cardinality of the set of signals does not pose any problem.

Second, we verify that the optimal disclosure policy in the class of symmetric and deterministic policies is indeed optimal in the general class of policies. To do so, we show that without loss of generality, one can bound the cardinality of $Z$ so that the problem becomes tractable, at least by numerical methods.

4.1. Symmetric and deterministic disclosure policies. A disclosure policy $(Z, \zeta)$ is said to be deterministic if it maps every outcome into a unique signal. That is, for any $\left(y_{A}, y_{B}\right) \in \mathcal{Y}$, there exists a signal $z \in Z$ such that $\zeta_{y_{A}, y_{B}}(z)=1$. A disclosure policy is said to be symmetric if for any signal $z \in Z$ there exists another signal $z^{\prime} \in Z$ (potentially, but not necessarily distinct from $z)$ such that $\zeta\left(z \mid y_{A}, y_{B}\right)=\zeta\left(z^{\prime} \mid y_{B}, y_{A}\right)$ for all $y_{A} \in\{s, f\}$ and $y_{B} \in\{s, f\}{ }^{11}$ That is, a symmetric disclosure policy treats the workers symmetrically ex ante: the "noise" in the disclosed information depends only on the relative performances of the workers, and not on the workers' identities.

Apart from simplifying the problem, there are two additional reasons for focusing on this class of policies. First, it is more realistic: it is perhaps more difficult for the firm to commit to a randomization mechanism over the signals. Also, symmetry implies that the firm is not biased towards any particular worker while choosing the level of noise it adds to its interim feedback. Second, it allows us to present an exposition of the trade-off between the effort levels in the two stages, and highlight the intuition behind the main result of the paper - the optimality of partial disclosure.

\footnotetext{
${ }^{10}$ Because the firm can observe only four events after the intermediate stage, we can restrict attention to disclosure policies with at most four signals if we assume that the firm cannot randomize. However, the firm might want to add some noise to the signal it sends, and if it is allowed to do that, it might want to choose a stochastic disclosure policy that assigns positive probability on more than four signals. Moreover, if the firm is allowed to randomize, it is not a priori obvious that we can restrict attention to disclosure policies with a finite number of signals.

${ }^{11}$ It is important to allow for the possibility that $z^{\prime}$ can be distinct from $z$, because requiring $z^{\prime}=z$ would rule out some interesting disclosure policies (e.g. full disclosure).
} 
There are seven policies in this class: (i) full disclosure, (ii) no disclosure, (iii) disclosing only if both succeed, (iv) disclosing only if both fail, (v) disclosing whether the scores are the same or not, (vi) disclosing whether the scores are the same, and if they are the same, whether both succeed or fail, and finally, (vii) disclosing who is the leader if the scores are different. The optimal disclosure policy is the one that induces the maximal expected aggregate effort over the two stages. Comparison of these policies leads to the following proposition:

Proposition 1. The optimal disclosure policy in the class of symmetric deterministic disclosure policies is to "disclose only if both fail." That is, the optimal disclosure policy is:

$$
Z=\left\{z_{1}, z_{2}\right\} \text {, where } \zeta_{f f}\left(z_{1}\right)=1 \text { and } \zeta_{s f}\left(z_{2}\right)=\zeta_{f s}\left(z_{2}\right)=\zeta_{s s}\left(z_{2}\right)=1 \text {. }
$$

Proposition 1 shows that the optimal disclosure policy uses two signals: one signal $\left(z_{1}\right)$ is revealed if both workers fail at the intermediate stage, and the other signal $\left(z_{2}\right)$ is revealed for all other outcomes at the intermediate stage.

To explain the intuition for this result, let us first consider the effect of disclosure policy on the final-stage effort (the ex-post effect) and on the intermediate-stage effort (the strategic effect) individually. Then we can analyze the interplay between the two effects and explain why the policy "disclose only if both fail" is optimal.

First, consider the ex-post effect of disclosure policy. To isolate this effect, we will assume that the intermediate-stage efforts are exogenous and equal to $e$. The first-order conditions (equations (Final) in the Appendix) imply that the total final-stage effort following any signal $z, E_{A}(z)+E_{B}(z)$, decreases in the probability (conditional on $z$ and $e$ ) that one of the workers is leading the race after the intermediate stage and increases in the probability (conditional on $z$ and $e$ ) that the scores are even. ${ }^{12}$ This suggests that, in order to achieve high aggregate final-stage effort, the disclosure policy should find the optimal balance between the tendency to reveal the fact the scores are even and the tendency to conceal the fact that the scores are uneven.

If the intermediate-stage efforts are exogenous and equal, all symmetric deterministic disclosure policies except for "full disclosure" result in the same expected aggregate effort. One way to understand this conclusion is to note that, for any disclosure policy in this class, the workers behave identically after any signal, since their beliefs about their relative positions are symmetric. Therefore the model becomes similar to a twice-replicated model with one worker. For such a model, one can prove that the expected marginal benefit of effort at the final stage and hence the expected final-stage effort is independent of the disclosure policy (see Lemma 1 in Lizzeri et al. (2002)).

Therefore, all symmetric deterministic disclosure policies except "full disclosure" result in the same expected aggregate effort, and the question of choosing the optimal policy boils down to the choice between "full disclosure" and any policy other than "full disclosure", such as "disclosing whether the score is even". The difference between these two policies is that under "full disclosure", when one of the workers is ahead after the first stage, the firm announces the identity of the winner whereas "disclosing whether the score is even" policy only informs the workers that the scores are uneven. Here, it is better to let the workers know the identity of the first-stage winner: the worker who knows for sure that he is leading will exert less effort than the one who knows he is behind, but the average of their efforts will be higher than the effort of a worker who knows the standing is uneven, but does not know who is leading. Therefore, "full disclosure" is optimal if there are no strategic effects of disclosure.

\footnotetext{
${ }^{12}$ This is a common feature of tournament models (see Lazear and Rosen, 1981; Rosen, 1986) and patent race games (see, e.g., Fudenberg et al.,1983, and Harris and Vickers, 1987).
} 
Next, let us consider the strategic effect of a disclosure policy. The first-order conditions for workers $A$ and $B$ with respect to their intermediate stage efforts are given by equation (4)). These conditions can be written as

$$
\begin{aligned}
& e_{B}\left(u_{A}^{s s}-u_{A}^{f s}\right)+\left(1-e_{B}\right)\left(u_{A}^{s f}-u_{A}^{f f}\right)=e_{A}, \\
& e_{A}\left(u_{B}^{s s}-u_{B}^{s f}\right)+\left(1-e_{A}\right)\left(u_{B}^{f s}-u_{B}^{f f}\right)=e_{B},
\end{aligned}
$$

where $u_{i}^{k l}$ is the expected payoff of worker $i$ at the final stage if the intermediate outcome is $\left(y_{A}=k, y_{B}=l\right) .{ }^{13}$ In a symmetric equilibrium, $u_{A}^{s s}-u_{A}^{f s}=u_{B}^{s s}-u_{B}^{s f}, u_{A}^{s f}-u_{A}^{f f}=u_{B}^{f s}-u_{B}^{f f}$, and one obtains

$$
e_{A}=e_{B}=\frac{u_{A}^{s f}-u_{A}^{f f}}{1+\left(u_{A}^{s f}-u_{A}^{f f}\right)-\left(u_{A}^{s s}-u_{A}^{f s}\right)} .
$$

So, in equilibrium, the intermediate-stage effort increases in the marginal benefit of effort when the opponent succeeds (i.e. $u_{A}^{s s}-u_{A}^{f s}$ ) as well as in the marginal benefit of effort when the opponent fails (i.e. $u_{A}^{s f}-u_{A}^{f f}$ ).

The optimal disclosure policy arises as a result of the interplay between the strategic and ex-post effects. If the intermediate-stage efforts are exogenously fixed at $e$, it is easy to verify that the expected final-stage effort under any symmetric deterministic disclosure policy is a decreasing function of $e$ for $e<0.5 .^{14}$ This suggests that the firm may face a trade-off between the strategic and ex-post effects: higher value of intermediate-stage effort will lead to higher probability of intermediate-stage success, which may decrease expected final-stage effort. This trade-off stems from the fact that policies that lead to high expected final-stage effort may affect the marginal gains in the final-stage expected payoffs, $u_{A}^{s s}-u_{A}^{f s}$ and $u_{A}^{s f}-u_{A}^{f f}$, so as to dampen the intermediate-stage effort incentives.

For example, let us contrast the policies of "no disclosure" and "disclose only if both succeed". As established above, if the intermediate-stage efforts under these policies were the same, the expected final-stage efforts would be the same as well. These two policies, however, affect the intermediate-stage effort incentives differently. Under the "no disclosure" policy the two stages are symmetric from the worker's point of view, therefore the effort is the same at both stages. In particular, $u_{A}^{s f}-u_{A}^{f f}=u_{A}^{s s}-u_{A}^{f s}=0.38$, so equation (6) yields $e_{A}=e_{B}=0.38$. Under the "disclose only if both succeed" policy, the gain in final stage payoff when the opponent fails, $u_{A}^{s f}-u_{A}^{f f}$, is slightly increased relative to "no disclosure"; however, the gain when the opponent succeeds, $u_{A}^{s s}-u_{A}^{f s}$, is significantly reduced. This is because succeeding if the opponent succeeds results in intense final stage competition, and, thus, relatively low expected final stage payoff. Therefore, in equilibrium, the marginal benefit of effort is lower than under the "no disclosure' policy, resulting in $e_{A}=e_{B}=0.363$.

Now we can compare the total effort under the two policies. As noted above, the expected final-stage effort is a decreasing function of the probability of intermediate-stage success. Therefore, lower intermediate-stage effort under "disclose only if both succeed" results in lower probability of success at the intermediate stage and thus higher expected final-stage effort. Indeed, under the "disclosure only if both succeed" policy, when both workers have succeeded, the race is revealed as tied, and both workers choose a high final-stage effort level of 0.5 ; when the workers know that at least one of them has failed, then both workers reduce

\footnotetext{
${ }^{13}$ For example, $u_{A}^{s s}=\sum_{z}\left[\frac{1}{2} E_{A} E_{B}+E_{A}\left(1-E_{B}\right)+\frac{1}{2}\left(1-E_{A}\right)\left(1-E_{B}\right)-\frac{1}{2} E_{A}^{2}\right] \zeta_{s s}(z)$. See equation (Int) in the Appendix for the expanded expression for (4).

${ }^{14}$ The final stage effort equals $\frac{1}{2}-\frac{2}{5} e+\frac{2}{5} e^{2}$ for "full disclosure" and $\frac{1}{2}\left(1-e+e^{2}\right)$ for all the other policies.
} 
their final-stage effort to 0.367 . The resulting expected final-stage effort equals 0.39 , versus 0.38 under "no disclosure." However, the increase in the expected final-stage effort under "disclose only if both succeed" is not enough to outweigh the negative strategic effect of this policy, and the total effort is lower than under "no disclosure."

The optimality of the "disclose only if both fail" policy stems from the trade-off discussed above. This policy, similar to the policy "disclose only if both succeed," induces intense competition by revealing information when the race is tied at $y_{A}=y_{B}=f$ and obscures the information about an uneven race by pooling these cases with the tied outcome $y_{A}=$ $y_{B}=s$. As a result, this policy leads to a very high marginal benefit of intermediate effort if the opponent fails (i.e., $u_{A}^{s f}-u_{A}^{f f}$ ). Indeed, failing if the opponent fails leads to intense final-stage competition, which results in low expected final-stage payoff. At the same time, the marginal benefit of intermediate effort if the opponent succeeds (i.e., $u_{A}^{s s}-u_{A}^{f s}$ ) is also relatively high. Succeeding if the opponent succeeds increases the likelihood of winning the tournament without engaging in an intense competition at the final stage. ${ }^{15}$ In effect, this policy punishes a worker for a lack of intermediate-stage effort by engaging him in an intense competition when both workers fail, and rewards the intermediate-stage effort by pooling the rest of the outcomes in just one message and thus making the final-stage race less competitive. Consequently, the "disclose only if both fail" policy enhances the marginal benefit of intermediate effort, leading to a high value of $e_{A}$ and $e_{B}$ : equation (6) yields $e_{A}=e_{B}=0.433$.

This policy, however, depresses the expected final-stage effort by reducing the probability that a tied race will be observed at the final stage. Because the intermediate efforts are high, it is less likely that both workers will fail, which is the only event where the workers learn that the race is tied, and hence, work intensely at the final stage. However, since the uneven race outcomes are pooled with the tied race outcome $y_{A}=y_{B}=s$, this policy continues to induce moderate effort levels even when the workers do not get any information about the intermediate outcome. As a result, the aggregate final expected effort $\mathbb{E}\left(E_{A}(z)+E_{B}(z)\right)$ reduces marginally (compared to the "no disclosure" case), whereas the intermediate effort increases significantly. These two effects taken together lead to a high aggregate effort. ${ }^{16}$

It turns out that the "disclose only if both fail" policy remains optimal even in the general class of disclosure policies. But before discussing such generalization, we highlight the implications of this finding.

4.1.1. Implications of the optimal disclosure policy. Proposition 1 implies that in our model it is in the interest of the firm neither to disclose the information about the intermediate performance fully, nor to conceal it entirely. Partial disclosure of performance feedback is a well-documented phenomenon: in hierarchical organizations, while rating the performance of their subordinates, supervisors often tend to use a coarse grading scale (Beer, 1990; Murphy, 1990; MacLeod, 2003). It is argued that such coarse grading - often referred to as "grade compression" - stems from the fact that supervisors face a substantial risk of straining their relationship with their subordinates if they provide harsh feedback (Beer, 1990; MacLeod, 2003). Our result suggests that partial disclosure can also occur in a dynamic tournament.

\footnotetext{
${ }^{15}$ Note that this feature is in sharp contrast with the "disclose only if both succeed" policy, where the likelihood of an intense competition in the final stage increases when both workers exert higher levels of intermediate effort.

${ }^{16}$ Similar reasoning suggests why the "disclose whether the score is even" policy is suboptimal. At the final stage, the workers compete intensively after they get the signal that their score is even. So this policy induces high effort at the final stage. However, at the intermediate stage, an increase in effort increases the probability of the outcome where both succeed, which leads to intense competition at the final stage. For that reason, this policy is dominated by the "disclose only if both fail" policy in terms of total effort, even though it generates higher effort at the final stage.
} 
However, rather than from the risk of straining relationship, it arises as a best response of the firm in order to resolve the trade-off between pre- and post-disclosure effort incentives.

This result is in contrast with the recent paper by Aoyagi (2010), which shows that one of the 'extreme' disclosure policies of full disclosure or no disclosure is always optimal. Therefore, it is important to draw out the factors that lead to this difference in findings. The key assumptions made by Aoyagi that differentiate his model from ours are: (i) a continuum of possible realizations of output at each stage; (ii) at each stage the firm can observe only the difference between the individual outputs and not their levels (in our model, that would translate into the assumption that the principal cannot distinguish between both agents succeeding and both failing); (iii) the difference in outputs at each stage is linear in the difference in efforts at that stage.

Aoyagi finds that the optimal disclosure policy is highly sensitive to the curvature of the workers' marginal cost of effort. If the marginal cost is convex, full disclosure is optimal in the class of all disclosure policies that have a symmetric equilibrium. If the marginal cost is concave, no disclosure is optimal, and if the cost function is quadratic (as it is assumed in our model), all disclosure policies induce the same expected effort. These results stem from the fact that the difference in outputs in each stage is linear in the difference in efforts. As a consequence, after any signal, both workers choose the same effort at the second stage. These efforts always cancel each other out, so it follows that the expected marginal benefit from increasing the effort at the second stage is the same for any disclosure policy. So the Jensen inequality implies that the expected final-stage effort is maximized by the no disclosure policy if the marginal cost of effort is convex, and by the full disclosure policy if the marginal cost is concave. Furthermore, it can be shown that the intermediate-stage effort is independent of the disclosure policy.

In contrast, in our model, effort affects the outcome distribution in a non-linear fashion. Moreover, the firm observes the intermediate-stage outputs of the workers and not just the difference between them. Consequently, the workers need not choose the same level of effort at the final stage, and the intermediate-stage efforts also vary with the firm's choice of the disclosure policy. Thus, Aoyagi's findings do not apply to our setting. Moreover, in contrast with Aoyagi (and also Ederer, 2010), we show that the disclosure policy of the firm does have an impact on the firm's payoff even when the workers' cost function is quadratic. In fact, in our model the optimal policy does not critically hinge on the curvature of the workers' cost function. ${ }^{17}$

Finally, our result suggests that conditioning the disclosure policy only on the difference between the workers' outputs (Yildirim, 2005; Aoyagi, 2010; Ederer, 2010) and/or imposing an a priori restriction by considering only the extreme policies of full disclosure or no disclosure (Lizzeri, et al., 2002; Ederer, 2010) may imply a loss of generality. The firm can be strictly better off by committing to a partial disclosure policy that is more nuanced than the extreme policies.

In the following subsection, we extend our analysis to include all feasible disclosure policies (not only symmetric and deterministic) and search for the optimal policy.

4.2. General disclosure policies. It is not obvious that the restriction to the class of symmetric and deterministic disclosure policy is without loss of generality. For example, it is not clear at the outset whether it is optimal for the firm to treat the workers the same way or to choose an asymmetric disclosure policy that favors one worker over another. An asymmetric policy may induce an asymmetric equilibrium where one worker exerts much

\footnotetext{
${ }^{17}$ As a suggestive evidence, we numerically solved the for the optimal policy under a more general cost function $e^{\gamma} / \gamma$. We took $\gamma \in[1.5,5]$ and found that the "disclose only if both fail" policy remains optimal for any such cost function.
} 
higher effort compared to the other worker, but the total effort is higher than the effort obtained under any symmetric policy. However, as we discuss below, the "disclose only if both fail" policy remains optimal even if more general disclosure policies are allowed.

In order to show this, we need to ensure that the general problem $\mathcal{P}$ is amenable to the standard optimization tools. As a first step, this requires bounding the number of signals to be used for disclosure. The following proposition is useful to overcome this problem.

Proposition 2. Consider a two-stage tournament with $n$ competitors and $k$ possible outcomes of the tournament at the intermediate stage. Then, if an optimal disclosure policy exists, there exists an optimal disclosure policy where $|Z| \leq n+k$.

Proposition 2 asserts that in our model with two workers and four intermediate outcomes $(\{s, s\},\{s, f\},\{f, s\},\{f, f\})$ one can restrict attention to the class of disclosure policies that maps the workers' performances into a set of signals that contains at most six elements. Thus the cardinality of the set of signals can be bounded from above so that the solution to the program $\mathcal{P}$ is tractable. ${ }^{18}$

Now, consider the problem $\mathcal{P}$. By Proposition 2, we can take $Z=\{1,2, \ldots, 6\}$ without loss of generality. This leaves us with the (IC) constraints and the condition that $\zeta$ must be a probability function on $Z$ for all $\left(y_{A}, y_{B}\right) \in \mathcal{Y}$ :

$$
\forall i, j \in\{s, f\}, \sum_{z=1}^{6} \zeta_{i j}(z)=1 \text { and } \zeta_{i j}(z) \geq 0 \forall z .
$$

The firm's problem can now be written as:

$$
\mathcal{P}^{\prime}\left\{\begin{array}{c}
\max _{\mathbf{e},\{\zeta(z), \mathbf{E}(z)\}_{z=1}^{6}} e_{A}+e_{B}+\sum_{\left(y_{A}, y_{B}\right) \in \mathcal{Y}} \operatorname{Pr}\left(y_{A}, y_{B}\right) \sum_{z=1}^{6}\left\{\left(E_{A}(z)+E_{B}(z)\right) \zeta_{y_{A} y_{B}}(z)\right\} \\
\text { s.t. (2), (4), and }(7) .
\end{array}\right.
$$

While Proposition 2 allows us to reduce the firm's problem to a tractable form given by $\mathcal{P}^{\prime}$, we still cannot apply the analytical optimization methods for finding the global maximum. This is due to the fact that the objective function is not concave in its arguments (after substituting in the expressions for $E_{A}(z)$ and $E_{B}(z)$ using (2), and writing the objective function as a function of $\left.\left(e_{A}, e_{B},\{\zeta(z)\}_{z=1}^{6}\right)\right)$. Thus, even if one can apply the standard optimization techniques to solve $\mathcal{P}^{\prime}$, it need not be the case that the solution obtained is a global maximum.

\footnotetext{
${ }^{18}$ The idea of the proof of Proposition 2 is as follows. Note that the only way the disclosure policy enters both the objective function and the constraints in the firm's problem $\mathcal{P}$ is through the expected value of the workers' effort level. However, if a probability distribution on some set has a certain expected value, it is possible to find another probability distribution on this set with the same expected value that assigns positive probabilities only to a finite number of mass points. Thus, we can restrict $Z$ to be a finite set without any loss of generality. But one can further bound the cardinality of the set $Z$. Suppose the optimal disclosure policy is $\left(Z^{*}, \zeta^{*}\right)$ and implements a profile of effort levels $\left\{\mathbf{e}^{*}, \mathbf{E}^{*}\right\}$. If so, then $\left(Z^{*}, \zeta^{*}\right)$ must also be a solution to an auxiliary problem of maximizing the firm's payoff by choosing a disclosure policy among the class of policies that implements $\left\{\mathbf{e}^{*}, \mathbf{E}^{*}\right\}$. Such an auxiliary problem can be formulated as a linear programming problem with $n+k$ nonnegativity constraints. The bound on $Z^{*}$ follows from determining the maximum number of choice variables in this problem that can take nonzero values at a corner solution.
} 
Confronting this issue, we solve the program by numerical methods. ${ }^{19}$ We find that the highest total effort under any disclosure policy is 1.621 , which is the same as the total effort under optimal symmetric deterministic policy "disclose only if both fail." This observation suggests that the policy "disclose only if both fail" is indeed optimal even in the most general class of disclosure policies.

\section{Discussion}

In this section we discuss the robustness of our findings to alternative modeling specifications. In particular, we explore whether the optimal disclosure policy is robust to variation in the size of the tournament rewards and alternative (asymmetric) tie-breaking rules. We also check whether the numerical optimization result of the previous section continues to hold with alternative production technologies. We discuss the role of the assumption that the firm can commit to a disclosure policy, and finally, explore the role of private disclosure policy in our model.

5.1. Robustness to variations in the tournament rewards. In the current version of the model, the firm cannot influence the post-promotion wages of the worker. However, whether the proposed disclosure policy is robust to a joint optimization over wages and feedback remains an interesting question. To address this issue, we depart from our model and assume that at the beginning of the tournament, the firm chooses the size of a reward pool, say $V$, and commits to allocate the reward between the winner and the loser.

Clearly, if $V$ is sufficiently large, then by promising $V$ to the winner and 0 to the loser, the firm can always implement the highest possible effort levels even without any feedback. Consequently, the question of optimal feedback becomes irrelevant. Hence, the question of joint optimization on reward and feedback is relevant only when there is some exogenous upper bound on the size of reward that the firm can offer. But even under this condition, one may expect the "disclose only if both fail" policy to remain optimal. Recall that the basic intuition behind the optimality of this policy is that it offers incentives at the intermediate stage without muting the final-stage incentives. At the intermediate stage, a success is rewarded since a successful worker is never made to face a stiff competition at the final stage, and failure is punished since a failed worker may face an intense race at the final stage. This argument does not hinge on the size of the reward.

\footnotetext{
${ }^{19}$ The technical details about the numerical optimization method are as follows. First, we substitute the expressions for $E_{A}$ and $E_{B}$ in the firm's objective function to rewrite the optimization problem as a problem of choosing twenty-six variables (two intermediate effort levels $\left(e_{A}, e_{B}\right)$, and twenty-four probability values representing four probability distributions, each corresponding to a particular realization of $\left(y_{A}, y_{B}\right)$, on the set of signals which has cardinality of six (i.e., $\left.\left\{\zeta_{s s}(z), \zeta_{f s}(z), \zeta_{s f}(z), \zeta_{f f}(z)\right\}_{z=1}^{6}\right)$ ), that maximizes the expected aggregate effort level subject to (4) and (7). The Matlab optimization routine we have used requires that the initial conditions must be feasible, so it is impossible to use equation (4) as an equality constraint (it is rarely possible to draw a tuple $\left(e_{A}, e_{B}\right)$ at random that satisfies (4)). Instead, a measure of the "error" due to not exactly satisfying constraint (4) is incorporated as a "penalty term" in the objective function. With this modification, the optimization routine can start with an arbitrary $\left(e_{A}, e_{B}\right)$ value but converges at a point that "almost" satisfies the equality constraint. More precisely, in order to solve $\min _{x} f(x)$ such that

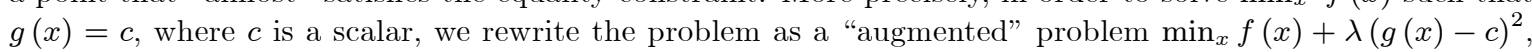
where $\lambda$ is a positive real number. The term $\lambda(g(x)-c)^{2}$ represents the "penalty" for not satisfying the equality constraint. This is always positive by construction, so the solution to the "augmented" problem must sufficiently minimize this penalty. Therefore, a solution to the "augmented" problem is a reasonable approximation of the solution to the original problem for a suitably chosen $\lambda$. We have used $\lambda=1000$ for all equality constraints in our program. The outcome of numerical optimization turned out to be highly dependent on the initial condition, so 100 simulations were performed, with initial values of these 26 variables drawn as a $26 \times 1$ vector from a uniform distribution in $[0,1]$.
} 
Given the non-linearity of the system of equations in (5), a complete analytical solution of the optimal disclosure policy is intractable for an arbitrary $V$. Hence, we numerically solve for the optimal disclosure policy. Figure 1(a) plots the payoff of the firm from each of the symmetric and deterministic disclosure policies as functions of the reward pool. Indeed, the results of numerical optimization suggest that the "disclose only if both fail" policy remains optimal for all relevant value of $V$.

This modification of the reward structure, however, does not allow for surplus destruction ( "money burning"). Depending on the outcome of the tournament, the firm can "destroy" a part of the prize pool so that neither the firm nor any of the workers get to keep it. Indeed, if money burning is credible, a whole array of more nuanced reward structures becomes feasible (see, e.g., Fuchs, 2007). A complete analysis of the optimal disclosure policy with money burning is beyond the scope of our paper. However, the trade-off between the pre- and post-disclosure effort levels continues to hold even in such an environment.
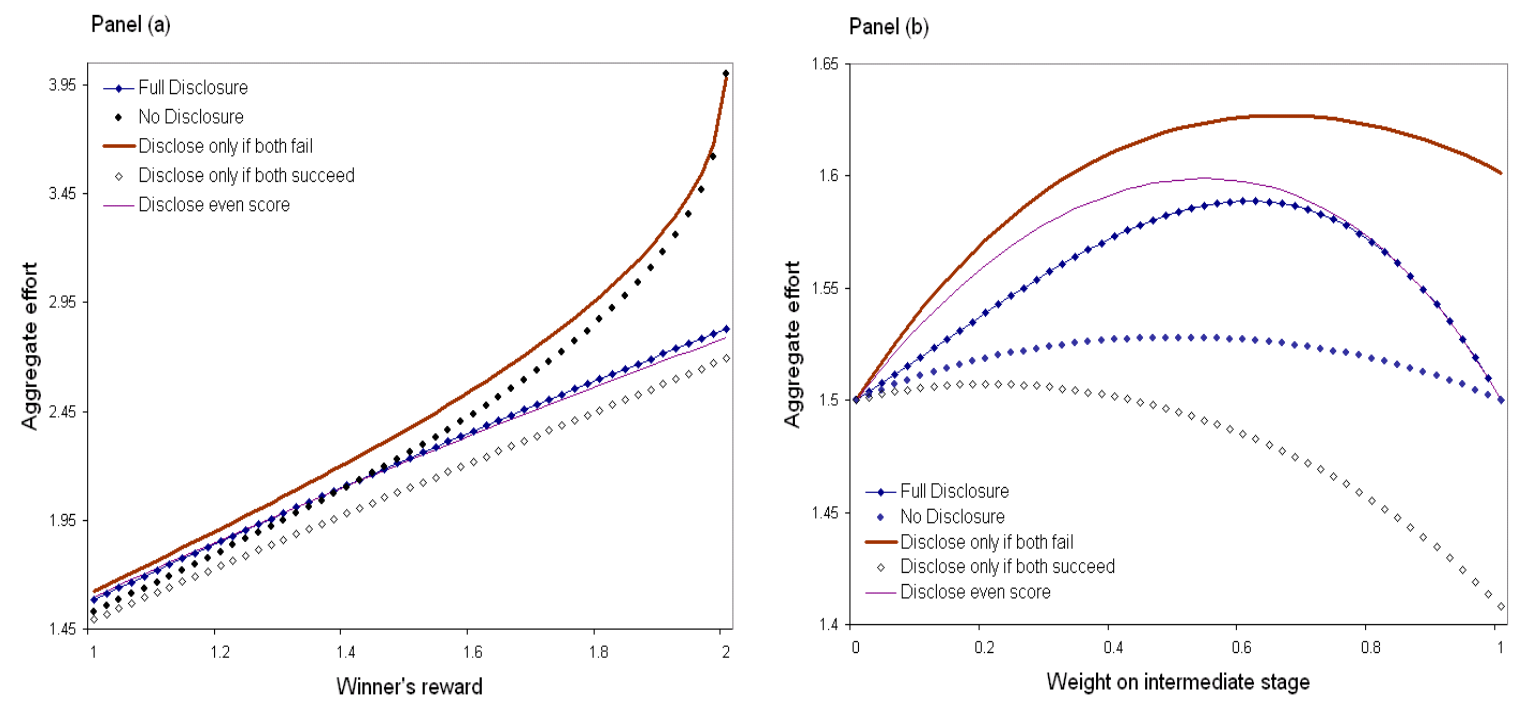

FiguRE 1. Firm's payoff from different disclosure policies as a function of winner's reward (panel (a)) and weight on the intermediate stage (panel (b))

5.2. Impact of alternative tie-breaking rules. Our model assumes a tie-breaking rule where a success in the intermediate stage is given the same weight as a success in the final stage. Consequently, if both workers have exactly one success, the tie is broken fairly between the workers irrespective of the stage in which the workers were successful.

But one may ask: does "disclose only if both fail" policy continue to remain optimal even if the firm weighs the outcomes of the two stages differently? Recall that the optimality of the "disclose only if both fail" policy is driven by the fact that this policy can shield a hard-working employee from future competitions but may expose a slacking worker to an intense competition in the final stage. In the process, it creates work incentives in the intermediate stage without completely blunting the final-stage effort incentives. But this argument continues to hold as long as there is some weight on the intermediate stage outcome. Thus, one may expect the "disclose only if both fail" policy to remain optimal even when the firm weighs the outcomes of the two stages differently. However, two issues remain: first, this argument does not indicate that putting equal weighs on the two stages is optimal. Second, as noted earlier, the high degree of non-linearity of the system of equations in (5) makes the 
model analytically intractable if one applies arbitrary weights on the outcomes of the two stages.

Hence, to investigate the robustness of our result to different weighting rules, we numerically solve for the optimal disclosure policy as a function of a tie-breaking probability $p$ that is defined as follows: if worker $i$ has one success in the intermediate stage, and worker $j$ one success in the final stage, then $i$ wins with probability $p$ and $j$ wins with probability $1-p$. If both workers have two successes or zero successes, the ties are broken with equal probabilities. That is, the smaller is the value of $p$, the less weight is given on the intermediate stage outcome. In the symmetric tie-breaking rule, we have $p=1 / 2$. Our numerical results shown in Figure 2(b) suggest that while it is not optimal to set $p=1 / 2$, for any value of $p$, "disclose only if both fail" remains the optimal disclosure policy.

5.3. Alternative production technologies. We further check for the robustness of our finding by considering the following variation to the model. Let the production technologies in the intermediate and the final stages be $\operatorname{Pr}\left(y_{i}=s\right)=\alpha e_{i}$ and $\operatorname{Pr}\left(Y_{i}=s\right)=\alpha E_{i}$, where $\alpha$ $\in(0,1]$ is a parameter that reflects the sensitivity of outcome with respect to the effort level. We keep all other aspects of the model unchanged. Note that we arrive at the original model by setting $\alpha=1$. For different values of $\alpha$, we obtain the firm's payoff associated with the numerical solution of the optimization problem $\mathcal{P}^{\prime}$ and compare it with the payoff associated with the disclosure policy "disclose only if both fail." Table 1 summarizes the results.

TABLE 1. Results of numerical optimization in the class of all feasible disclosure policies

\begin{tabular}{|c|c|c|c|c|}
\hline \multirow[t]{2}{*}{$\alpha$} & \multicolumn{3}{|c|}{$\begin{array}{l}\text { Effort under "disclose only if } \\
\text { both fail" policy }\end{array}$} & \multirow{2}{*}{$\begin{array}{c}\begin{array}{c}\text { Effort under optimal policy in } \\
\text { general class of policies }\end{array} \\
\text { Aggregate effort }\end{array}$} \\
\hline & $e_{A}+e_{B}$ & $\mathbb{E}\left(E_{A}+E_{B}\right)$ & Aggregate effort & \\
\hline 1.0 & 0.867 & 0.754 & 1.621 & 1.621 \\
\hline 0.8 & 0.737 & 0.634 & 1.371 & 1.371 \\
\hline 0.6 & 0.578 & 0.514 & 1.092 & 1.092 \\
\hline 0.4 & 0.395 & 0.371 & 0.766 & 0.766 \\
\hline 0.2 & 0.199 & 0.196 & 0.396 & 0.396 \\
\hline
\end{tabular}

For different values of $\alpha$, Table 1 shows the aggregate effort (and the associated levels of intermediate-stage and expected final-stage efforts) obtained from the optimal symmetric deterministic disclosure policy and the aggregate effort associated with the optimal policy in the general class of policies (obtained from solving $\mathcal{P}^{\prime}$ numerically). Comparing the two sets of data, it is evident that the policy "disclose only if both fail" remains optimal even if one perturbs the model by varying the production technology through the parameter $\alpha$.

5.4. Commitment to a disclosure policy. An important assumption in our model is that the firm can commit to a disclosure policy. If the firm cannot commit to a disclosure policy, then the feedback becomes "cheap talk", and the firm will always find it profitable to send the feedback that leads to the highest aggregate effort in the final stage. Consequently, in equilibrium the workers will treat the feedback as pure noise, and choose their efforts independently of the signal revealed. In other words, commitment to a disclosure policy is crucial in our setting for such policy to have any incentive implications.

The commitment assumption is a natural starting point for the study of optimal feedback but it is a strong assumption when the performance measure is subjective. However, the 
firm can credibly implement a disclosure policy in several ways. For example, in many client service industries the workers can infer about their performance based on the clients' reaction, and the firm can control the extent of interaction between its workers and clients (through appropriate job design) in order to filter the information feedback. ${ }^{20}$ Similarly, in many cases, performance feedback is based on evaluations from multiple sources (e.g., the so-called $360^{0}$ feedback, where the worker is reviewed not only by his supervisor but also by his subordinates). The worker may be able to cross check the feedback from the supervisor with the information available from other sources. Consequently, there is limited scope for the firm to lie in its feedback. A formal analysis of the optimal disclosure policy in absence of any commitment power is beyond the scope of our model but it remains an interesting open question for future research.

5.5. Private feedback. Our analysis solely focuses on public disclosure policies where at the end of the intermediate stage, both workers publicly observe the same signal. However, private feedback policies where employees only know their evaluations and not those of their peers are indeed common in many settings. Does the policy "disclose only if both fail" remain optimal if private disclosure policies are feasible?

The answer to this question depends on the class of private feedback policies that the firm can commit to. Note that our commitment assumption becomes more tenuous in the case of private feedback since it might be difficult for a third party to verify any privately communicated signal. If the firm can only commit to private disclosure policies where the worker is privately given information only on his own performance and not on that of his competitors, then the optimal public policy "disclose only if both fail" cannot be replicated by any private feedback policy. This class of private feedback policies contains only two symmetric deterministic policies: (i) neither worker gets any information about his outcome and (ii) each worker gets to know with certainty whether he has succeeded or failed in the intermediate stage. But both of these policies are worse than "disclose only if both fail" policy. Policy (i) is equivalent to no disclosure, and policy (ii) results in an aggregate expected effort that equals to 1.576, which is lower than the effort resulting from the policy "disclose only if both fail."

However, if the firm can commit to a private feedback policy that is informative of the relative performance of the workers, then the firm can do strictly better than the "disclose only if both fail" policy. For example, suppose that, if a worker fails, the firm privately informs him of both his own and the competitor's intermediate-stage outcome, and if a worker succeeds, gives him no information. The total expected effort under this policy is 1.622 , slightly higher than what can be implemented by the optimal public policy. ${ }^{21}$ The example above suggests that even if the firm can commit to private disclosure policies, partial disclosure outperforms the extreme policies of full and no disclosure. However, a complete analytical characterization of the optimal disclosure policy if private signals are allowed is beyond the scope of this article.

\section{Conclusion}

This paper offers a stylized model of a multistage tournament where the workers compete through two stages. When the firm privately observes the workers' performances in each stage of the tournament, the firm faces the choice of how much information to reveal to the workers at the interim stage in order to maximize their effort incentives. We argue that the optimal disclosure policy must resolve the following trade-off: the disclosure policy that

\footnotetext{
${ }^{20}$ For example, in some cases information technology firms can choose whether to send their engineers to the client site or to work on their own facility and ship out the final product. By making such a choice, the firm can manipulate the extent of client contact with its workers (see Loveman and O'Connell, 1996).

${ }^{21}$ The computation of optimal effort is similar to the ones done in the proof of Proposition 1 in the Appendix.
} 
motivates the workers to exert high effort at the final stage can dampen the incentives at the intermediate stage.

The optimal disclosure policy in our model takes a simple form: the firm reveals information (without any noise) only if both workers do poorly at the intermediate stage, and does not reveal any information otherwise. This result provides a novel justification for the optimality of partial disclosure, which is a well-documented feature of interim feedback in hierarchical organizations. This result is also in sharp contrast with the existing literature that suggests that one of the 'extreme' policies of full disclosure and no disclosure is likely to be the optimal policy.

The key contribution of this paper is to highlight the trade-off between the pre- and postdisclosure effort incentives that leads the firm to filter the performance feedback it offers to its employees. But firms offer performance feedback for several reasons, and enhancing work incentives is just one of them. For example, based on the feedback, a worker can identify skills that he needs to develop, and consequently, can undertake efficient investments in human capital (Beer, 1987). In a more general setting, the intermediate-stage outcome reveals the worker's ability with some noise, so that the workers also gradually learn about each others' abilities from the feedback (Ederer, 2010). How any of these issues would influence the optimal disclosure policy can be an interesting question for the future research. However, even in such complex settings the trade-off between pre- and post-disclosure incentives is likely to play a significant role in governing the firm's choice of the disclosure policy.

\section{APPENDIX}

A. Proofs omitted in the text. Before we present the proofs, it is useful to expand on the constraints on the intermediate and final-stage efforts in the firm's optimization problem $\mathcal{P}^{\prime}$. For every $z \in Z$, let $P_{y_{A} y_{B}}(z)=\operatorname{Pr}\left(y_{A}, y_{B} \mid z\right)$ be the posterior probability of intermediate outcome $\left(y_{A}, y_{B}\right)$ conditional on signal $z$ being observed. For example, $\operatorname{Pr}\left(y_{A}=s, y_{B}=s \mid z\right) \equiv P_{s s}(z)$, where

$$
P_{s s}(z)=\frac{\zeta_{s s}(z) e_{A} e_{B}}{\zeta_{s s}(z) e_{A} e_{B}+\zeta_{s f}(z) e_{A}\left(1-e_{B}\right)+\zeta_{f s}(z)\left(1-e_{A}\right) e_{B}+\zeta_{f f}(z)\left(1-e_{A}\right)\left(1-e_{B}\right)} .
$$

The terms $P_{s f}, P_{f s}$ and $P_{f f}$ are defined similarly. ${ }^{22}$ Then condition (1) can be rewritten as

$$
\begin{aligned}
& E_{i}^{*}(z)= \\
& \begin{aligned}
\arg \max _{\hat{E}_{i} \in[0,1]} \hat{E}_{i}\left(1-E_{j}(z)\right)\left(P_{s s}(z)+P_{f f}(z)\right)+ & \left(\hat{E}_{i}+\left(1-\hat{E}_{i}\right)\left(1-E_{j}(z)\right)\right) P_{\alpha \beta}(z) \\
+\frac{1}{2}\left[( \hat { E } _ { i } E _ { j } ( z ) + ( 1 - \hat { E } _ { i } ) ( 1 - E _ { j } ( z ) ) ) \left(P_{s s}(z)+\right.\right. & \left.P_{f f}(z)\right)+\left(E_{j}(z)\left(1-\hat{E}_{i}\right)\right) P_{\alpha \beta}(z) \\
& \left.+\left(\hat{E}_{i}\left(1-E_{j}(z)\right)\right) P_{\beta \alpha}(z)\right]-\frac{1}{2} \hat{E}_{i}^{2},
\end{aligned}
\end{aligned}
$$

where $\alpha=s$ if $i=A$ and $f$ otherwise, and $\beta=s$ if $i=B$ and $f$ otherwise. If there exists an interior solution to problem (1), the first-order conditions for this problem are:

$$
\begin{aligned}
& E_{A}(z)=\frac{1}{2} P_{s s}(z)+\frac{1}{2} P_{s f}(z) E_{B}(z)+\frac{1}{2} P_{f s}(z)\left(1-E_{B}(z)\right)+\frac{1}{2} P_{f f}(z), \\
& E_{B}(z)=\frac{1}{2} P_{s s}(z)+\frac{1}{2} P_{f s}(z) E_{A}(z)+\frac{1}{2} P_{s f}(z)\left(1-E_{A}(z)\right)+\frac{1}{2} P_{f f}(z) .
\end{aligned}
$$

Solving for $E_{A}(z)$ and $E_{B}(z)$ yields:

(Final)

$$
\begin{aligned}
& E_{A}(z)=\frac{1}{2} \frac{P_{s s}(z)+P_{f s}(z)+P_{f f}(z)+\frac{1}{2}\left(P_{s f}(z)-P_{f s}(z)\right)\left(P_{s s}(z)+P_{s f}(z)+P_{f f}(z)\right)}{1+\frac{1}{4}\left(P_{f s}(z)-P_{s f}(z)\right)^{2}}, \\
& E_{B}(z)=\frac{1}{2} \frac{P_{s s}(z)+P_{s f}(z)+P_{f f}(z)+\frac{1}{2}\left(P_{f s}(z)-P_{s f}(z)\right)\left(P_{s s}(z)+P_{f s}(z)+P_{f f}(z)\right)}{1+\frac{1}{4}\left(P_{f s}(z)-P_{s f}(z)\right)^{2}} .
\end{aligned}
$$

\footnotetext{
${ }^{22}$ We suppress the $e_{i}$ s in the notation for $P_{y_{A} y_{B}}(z)$ for the sake of expositional clarity. Also, without loss of generality, we will consider only those signals $z$ for which $P_{y_{A} y_{B}}(z)>0$.
} 
Note that $E_{i}(z) \mathrm{s}$ will also depend on $e_{i} \mathrm{~s}$ through their effect on $P_{y_{A} y_{B}}(z) \mathrm{s}$. Next, note that if there exists an interior solution to problem (3), the first-order conditions (4) for the intermediate effort, $e_{A}$ and $e_{B}$, are: ${ }^{23}$

(Int)

$$
\begin{aligned}
e_{i}= & e_{j} \sum_{z} \frac{1}{2}\left[E_{i}(z) E_{j}(z)+\left(E_{i}(z)+\frac{1}{2}\left(1-E_{i}(z)\right)\right)\left(1-E_{j}(z)\right)-\frac{1}{2} E_{i}^{2}(z)\right] \zeta_{s s}(z) \\
& +\left(1-e_{j}\right) \sum_{z}\left[1-\frac{1}{2} E_{j}(z)\left(1-E_{i}(z)\right)-\frac{1}{2} E_{i}^{2}(z)\right] \zeta_{\alpha \beta}(z) \\
& -e_{j} \sum_{z}\left[\frac{1}{2} E_{i}(z)\left(1-E_{j}(z)\right)-\frac{1}{2} E_{i}^{2}(z)\right] \zeta_{\beta \alpha}(z) \\
& -\left(1-e_{j}\right) \sum_{z}\left[\frac{1}{2} E_{i}(z) E_{j}(z)+\left(E_{i}(z)+\frac{1}{2}\left(1-E_{i}(z)\right)\right)\left(1-E_{j}(z)\right)-\frac{1}{2} E_{i}^{2}(z)\right] \zeta_{f f}(z),
\end{aligned}
$$

where $\alpha=s$ if $i=A$ and $f$ otherwise, and $\beta=s$ if $i=B$ and $f$ otherwise. Therefore, the firm's problem $\mathcal{P}^{\prime}$ can now be written as:

$$
\mathcal{P}^{\prime}\left\{\begin{array}{l}
\max _{\left\{\mathbf{e},\{\zeta(z), \mathbf{E}(z)\}_{z=1}^{6}\right\}} e_{A}+e_{B}+\sum_{\left(y_{A}, y_{B}\right) \in \mathcal{Y}} \operatorname{Pr}\left(y_{A}, y_{B}\right) \sum_{z=1}^{6}\left\{\left(E_{A}(z)+E_{B}(z)\right) \zeta_{y_{A} y_{B}}(z)\right\} \\
\text { s.t. }(\text { Int }),(\text { Final }), \text { and } \forall i, j \in\{s, f\}, \sum_{z=1}^{6} \zeta_{i j}(z)=1 \text { where } \zeta_{i j}(z) \geq 0 \forall z .
\end{array}\right.
$$

We now present the proofs omitted in the text.

Proof of Lemma 1. Given a disclosure policy $(Z, \zeta)$, we will prove that there exists a pair of values of intermediate effort levels $\left(e_{i}\right)$ and a pair of mappings $E_{i}: Z \rightarrow \mathbb{R}$ for $i \in\{A, B\}$ such that the tuple $\left\{e_{i}, E_{i}(z)\right\}$ satisfies the conditions (Final) and (Int), and both $e_{i}$ and $E_{i}(z)$ are in $[0,1]$ for all values of $z$. Since problems $(1)$ and $(3)$ are concave, this is sufficient to conclude that the tuple $\left\{e_{i}, E_{i}(z)\right\}$ constitutes a PBE of the tournament game between the workers. We present the proof in the following steps.

Step 1. First, we prove that $0 \leq E_{i}(z)<1$ for any $z \in Z$, where $E_{i}(z)$ are defined by (Final). For any $z \in Z$,

$$
\begin{aligned}
E_{A}(z) & =\frac{1}{2} \frac{P_{s s}(z)+P_{f s}(z)+P_{f f}(z)+\frac{1}{2}\left(P_{s f}(z)-P_{f s}(z)\right)\left(P_{s s}(z)+P_{s f}(z)+P_{f f}(z)\right)}{1+\frac{1}{4}\left(P_{f s}(z)-P_{s f}(z)\right)^{2}} \\
& \leq \frac{1}{2}\left[P_{s s}(z)+P_{f s}(z)+P_{f f}(z)+\frac{1}{2}\left(P_{s f}(z)-P_{f s}(z)\right)\left(P_{s s}(z)+P_{s f}(z)+P_{f f}(z)\right)\right] .
\end{aligned}
$$

But recall that $P_{y_{A} y_{B}}$ is a probability distribution over the set $\mathcal{Y}$. Thus, for all $\left(y_{A}, y_{B}\right)$, $P_{y_{A} y_{B}} \in[0,1]$, and $\sum P_{y_{A} y_{B}}=1$. So, each of the following terms, $P_{s s}(z)+P_{f s}(z)+P_{f f}(z)$, $P_{s f}(z)-P_{f s}(z)$, and $P_{s s}(z)+P_{s f}(z)+P_{f f}(z)$ are bounded above by 1 . Setting each of these three terms to 1 , we obtain $E_{A}(z) \leq 3 / 4$. Further note that

$$
\begin{aligned}
& \operatorname{sign} E_{A}(z)= \\
& \operatorname{sign}\left[P_{s s}(z)+P_{f s}(z)+P_{f f}(z)+\frac{1}{2}\left(P_{s f}(z)-P_{f s}(z)\right)\left(P_{s s}(z)+P_{s f}(z)+P_{f f}(z)\right)\right] .
\end{aligned}
$$

But,

$$
\begin{aligned}
& P_{s s}(z)+P_{f s}(z)+P_{f f}(z)+\frac{1}{2}\left(P_{s f}(z)-P_{f s}(z)\right)\left(P_{s s}(z)+P_{s f}(z)+P_{f f}(z)\right)= \\
& \left(1-P_{s f}(z)\right)+\frac{1}{2}\left(P_{s f}(z)-P_{f s}(z)\right)\left(1-P_{f s}(z)\right) \geq 0,
\end{aligned}
$$

where the last inequality holds as both $P_{s f}$ and $P_{f s}(z)$ are in $[0,1]$ and $P_{s f}(z)+P_{f s}(z) \leq 1$. Hence, $E_{A}(z) \geq 0$.

By similar argument, $0 \leq E_{B}(z) \leq 3 / 4, \forall z \in Z$.

Step 2. Next, we show that equations (Int) have a solution $\left(e_{A}, e_{B}\right) \in[0,1]^{2}$. Let $f_{A}\left(e_{A}, e_{B}\right)$ and $f_{B}\left(e_{A}, e_{B}\right)$ denote the right-hand side of the first and the second equation of (Int),

\footnotetext{
${ }^{23}$ Note that $E_{i}(z)$ also depend on $e_{i}$. However, these effects are zero by the envelope theorem and therefore omitted in the equations (Int).
} 
respectively, where $\left\{E_{A}(z), E_{B}(z)\right\}_{z \in Z}$ are considered as functions of $\left(e_{A}, e_{B}\right)$ and determined by equations (Final). Both $f_{A}\left(e_{A}, e_{B}\right)$ and $f_{B}\left(e_{A}, e_{B}\right)$ are continuous. Moreover, if $\left(e_{A}, e_{B}\right) \in[0,1]^{2}$, then $\left(f_{A}\left(e_{A}, e_{B}\right), f_{B}\left(e_{A}, e_{B}\right)\right) \in[0,1]^{2}$. To see this, note that we can rewrite $f_{A}$ as:

$$
\begin{aligned}
f_{A}\left(e_{A}, e_{B}\right)= & e_{B} \int K_{s s}(z) d \zeta_{s s}(z)+\left(1-e_{B}\right) \int K_{s f}(z) d \zeta_{s f}(z)-e_{B} \int K_{f s}(z) d \zeta_{f s}(z) \\
& -\left(1-e_{B}\right) \int K_{f f}(z) d \zeta_{f f}(z),
\end{aligned}
$$

where we define

$$
\begin{aligned}
K_{s s} & :=\frac{1}{2} E_{A} E_{B}+E_{A}\left(1-E_{B}\right)+\frac{1}{2}\left(1-E_{A}\right)\left(1-E_{B}\right)-\frac{1}{2} E_{A}^{2} \\
K_{s f} & :=E_{A} E_{B}+E_{A}\left(1-E_{B}\right)+\left(1-E_{A}\right)\left(1-E_{B}\right)+\frac{1}{2}\left(1-E_{A}\right) E_{B}-\frac{1}{2} E_{A}^{2} \\
K_{f s} & :=\frac{1}{2} E_{A}-\frac{1}{2} E_{A} E_{B}-\frac{1}{2} E_{A}^{2} \\
K_{f f} & :=K_{s s}(z) .
\end{aligned}
$$

Now, using the fact that $\left(E_{A}(z), E_{B}(z)\right) \in[0,3 / 4]^{2}$, we obtain $K_{s s} \in[1 / 8,5 / 8], K_{s f} \in$ [5/8,1], $K_{f s} \in[-3 / 64,1 / 8]$, and $K_{s s} \in[1 / 8,5 / 8]$. Thus, one can bound $f_{A}$ as follows:

$$
f_{A}\left(e_{A}, e_{B}\right) \leq 5 e_{B} / 8+\left(1-e_{B}\right)+3 e_{B} / 64-\left(1-e_{B}\right) / 8<1,
$$

and

$$
f_{A}\left(e_{A}, e_{B}\right) \geq e_{B} / 8+5\left(1-e_{B}\right) / 8-e_{B} / 8-5\left(1-e_{B}\right) / 8=0 .
$$

Similarly, $f_{B}\left(e_{A}, e_{B}\right) \in[0,1]$ if $\left(e_{A}, e_{B}\right) \in[0,1]^{2}$. By Brouwer's fixed-point theorem, it follows that the function $\left(f_{A}, f_{B}\right):[0,1]^{2} \rightarrow[0,1]^{2}$ has a fixed point, which defines equilibrium intermediate effort levels.

Proof of Proposition 1. The proof is given in the following steps:

Step 1. Observe the following.

(a) Policy (v) ("disclosing whether the score is the same or not") generates the same payoff for the firm as policy (vi) ("disclosing whether the score is the same, and if it is the same, whether both succeed or fail"). This is because a signal $z$ such that $\zeta_{s s}(z)=$ $\zeta_{f f}(z)=1, \zeta_{f s}(z)=\zeta_{s f}(z)=0$ leads to the same final-stage effort as a signal $z^{\prime}$ such that $\zeta_{s s}\left(z^{\prime}\right)=1, \zeta_{f f}\left(z^{\prime}\right)=\zeta_{s f}\left(z^{\prime}\right)=\zeta_{f s}\left(z^{\prime}\right)=0$, or a signal $z^{\prime \prime}$ such that $\zeta_{f f}\left(z^{\prime \prime}\right)=1$, $\zeta_{s s}\left(z^{\prime \prime}\right)=\zeta_{s f}\left(z^{\prime \prime}\right)=\zeta_{f s}\left(z^{\prime \prime}\right)=0$ (namely, the final-stage effort is $1 / 2$ for both participants following any of these signals). Consequently, policy (v) generates the same final-stage efforts as policy (vi), and thus, also the lead to the same intermediate-stage efforts. In words, it does not matter whether only to disclose that the score is even, or to disclose the actual outcome, given that the score is even.

(b) policy (i) ("full disclosure") generates the same payoff for the firm as policy (vii) ("disclosing who is the leader if the score is not the same"). The argument is the same as above.

Step 2. Given this observation, we effectively have only five different cases. We compute the firm's payoff in each of these five cases as follows:

(i) Full disclosure: $Z=\left\{z_{s s}, z_{s f}, z_{f s}, z_{f f}\right\}, \zeta_{y_{A} y_{B}}\left(z_{y_{A} y_{B}}\right)=1$.

Here $P_{y_{A} y_{B}}\left(z_{y_{A}, y_{B}}\right)=1$, so substituting into (Final) yields $E_{A}\left(z_{s s}\right)=E_{B}\left(z_{s s}\right)=E_{A}\left(z_{f f}\right)=$ $E_{B}\left(z_{f f}\right)=1 / 2 ; E_{A}\left(z_{s f}\right)=E_{B}\left(z_{f s}\right)=1 / 5$, and $E_{A}\left(z_{f s}\right)=E_{B}\left(z_{s f}\right)=2 / 5$. Substituting these values into (Int) we solve for the intermediate-stage effort level $e_{A}=e_{B}=89 / 230 \approx$ 0.38696 . Using the equilibrium value of $e_{i}$, we obtain $\mathbb{E}\left(E_{A}(z)+E_{B}(z)\right)=0.81022$. Thus the expected total effort is $2 e_{A}+\mathbb{E}\left(E_{A}(z)+E_{B}(z)\right) \approx 1.5841$.

(ii) No disclosure: $Z=\{z\}, \zeta_{y_{A} y_{B}}(z)=1$. 
With no disclosure, the two stages are symmetric, so $e_{A}=e_{B}=E_{A}=E_{B}=e$. Substituting into (Final) and solving for $e$ yields $e=(3-\sqrt{5}) / 2$. Thus, the total effort is $4 e=2(3-\sqrt{5}) \approx 1.5279$.

(iii) Disclosing only if both succeed: $Z=\left\{z_{1}, z_{2}\right\}, \zeta_{s s}\left(z_{1}\right)=1, \zeta_{s f}\left(z_{2}\right)=\zeta_{f_{s}}\left(z_{2}\right)=$ $\zeta_{f f}\left(z_{2}\right)=1$.

At the final stage, the effort after signal $z_{1}$ is $E_{A}\left(z_{1}\right)=E_{B}\left(z_{1}\right)=1 / 2$. After signal $z_{2}$, in a symmetric equilibrium the posteriors are $P_{s s}\left(z_{2}\right)=0, P_{s f}\left(z_{2}\right)=P_{f s}\left(z_{2}\right)=$ $e(1-e) /\left(1-e^{2}\right)$, and $P_{f f}\left(z_{2}\right)=(1-e)^{2} /\left(1-e^{2}\right)$. So $E_{A}\left(z_{2}\right)=E_{B}\left(z_{2}\right)=$ $\frac{1}{2}\left(P_{s s}\left(z_{2}\right)+P_{f s}\left(z_{2}\right)+P_{f f}\left(z_{2}\right)\right)=1 / 2(1+e)$. At the intermediate stage,

$$
\begin{aligned}
e & =\frac{3}{8} e+(1-e)\left(1-\frac{1}{2} E_{A}\left(z_{2}\right)\right)-e\left(\frac{1}{2} E_{A}\left(z_{2}\right)-\left(E_{A}\left(z_{2}\right)\right)^{2}\right)-(1-e)\left(\frac{1}{2}-\frac{1}{2}\left(E_{A}\left(z_{2}\right)\right)^{2}\right) \\
& =e\left(\frac{1}{2}\left(E_{A}\left(z_{2}\right)\right)^{2}-\frac{1}{8}\right)+\frac{1}{2}\left(E_{A}\left(z_{2}\right)\right)^{2}-\frac{1}{2} E_{A}\left(z_{2}\right)+\frac{1}{2} .
\end{aligned}
$$

Substituting for $E_{A}\left(z_{2}\right)$, we get $9 e_{A}^{3}+14 e_{A}^{2}+2 e_{A}-3=0$. The only solution that lies in [0,1] is $e \approx 0.3629$ (one can check that this solution for $e$ satisfies the second-order conditions). Thus, the expected final-stage effort is $\mathbb{E}_{(}\left(E_{A}(z)+E_{B}(z)\right)=1-e+e^{2} \approx 0.7688$. The resulting total effort is $2 e+\mathbb{E}\left(E_{A}(z)+E_{B}(z)\right) \approx 1.4946$. 1 .

(iv) Disclosing only if both fail: $Z=\left\{z_{1}, z_{2}\right\}, \zeta_{f f}\left(z_{1}\right)=1, \zeta_{s f}\left(z_{2}\right)=\zeta_{f s}\left(z_{2}\right)=\zeta_{s s}\left(z_{2}\right)=$

At the final stage, the effort after signal $z_{1}$ is $E_{A}\left(z_{1}\right)=E_{B}\left(z_{1}\right)=1 / 2$. After signal $z_{2}$, in a symmetric equilibrium the posteriors are $P_{f f}\left(z_{2}\right)=0, P_{s f}\left(z_{2}\right)=P_{f s}\left(z_{2}\right)$ $=e(1-e) /\left(1-(1-e)^{2}\right), P_{s s}\left(z_{2}\right)=e^{2} /\left(1-(1-e)^{2}\right)$. So $E_{A}\left(z_{2}\right)=E_{B}\left(z_{2}\right)=1 / 2(2-e)$. At the intermediate stage,

$$
\begin{aligned}
e & =e\left(\frac{1}{2}-\frac{1}{2}\left(E_{A}\left(z_{2}\right)\right)^{2}\right)+(1-e)\left(1-\frac{1}{2} E_{A}\left(z_{2}\right)\right)-e\left(\frac{1}{2} E_{A}\left(z_{2}\right)-\left(E_{A}\left(z_{2}\right)\right)^{2}\right)-\frac{3}{8}(1-e) \\
& =e\left(\frac{1}{2}\left(E_{A}\left(z_{2}\right)\right)^{2}-\frac{1}{8}\right)-\frac{1}{2} E_{A}\left(z_{2}\right)+\frac{5}{8} .
\end{aligned}
$$

Substituting for $E_{A}\left(z_{2}\right)$ and reorganizing yields $9 e^{3}-41 e^{2}+53 e-16=0$. The only solution that lies in $[0,1]$ is $e \approx 0.4333$ (one can check that this solution for $e$ satisfies the second-order conditions). Thus, the expected final-stage effort is $\mathbb{E}\left(E_{A}(z)+E_{B}(z)\right)=1-e+e^{2} \approx 0.7544$. The resulting total effort is $2 e+\mathbb{E}\left(E_{A}(z)+E_{B}(z)\right) \approx 1.6211$.

(v) Disclosing whether the score is even: $Z=\left\{z_{1}, z_{2}\right\}, \zeta_{s s}\left(z_{1}\right)=\zeta_{f f}\left(z_{1}\right)=1, \zeta_{s f}\left(z_{2}\right)=$ $\zeta_{f s}\left(z_{2}\right)=1$.

After signal $z_{1}$, in a symmetric equilibrium the posteriors are $P_{f f}\left(z_{1}\right)=$ $(1-e)^{2} /\left(e^{2}+(1-e)^{2}\right), P_{s s}\left(z_{1}\right)=e^{2} /\left(e^{2}+(1-e)^{2}\right), P_{s f}\left(z_{1}\right)=P_{f s}\left(z_{1}\right)=0$. The finalstage efforts after signal $z_{1}$ are $E_{A}\left(z_{1}\right)=E_{B}\left(z_{1}\right)=1 / 2$. After signal $z_{2}$, in a symmetric equilibrium the posteriors are $P_{f f}\left(z_{2}\right)=P_{s s}\left(z_{2}\right)=0$, and $P_{s f}\left(z_{2}\right)=P_{f s}\left(z_{2}\right)=1 / 2$. The final-stage efforts after signal $z_{2}$ are $E_{A}\left(z_{2}\right)=E_{B}\left(z_{2}\right)=1 / 4$. Substituting the values of $E_{A} \mathrm{~S}$ and $E_{B} \mathrm{~S}$ into (Int), we can solve for $e \approx 0.4211$. Thus, the expected final-stage effort is $\mathbb{E}\left(E_{A}(z)+E_{B}(z)\right) \approx 0.7562$, and the resulting total effort is $2 e+\mathbb{E}\left(E_{A}(z)+E_{B}(z)\right) \approx$ 1.598 .

Thus, the highest total effort is obtained under the policy "disclosing only if both fail."

Proof of Proposition 2. We will present the proof in the context of a model with $n$ players and $k$ possible intermediate outcomes, $y \in\left\{y_{1}, \ldots, y_{k}\right\}$. The proof is given in the following steps:

Step 1. Fix a disclosure policy $(Z, \zeta)$. Let $Z_{i} \subseteq Z$ be the subset of signals that are in the support of $\zeta_{y_{i}}$, but not in the support of $\zeta_{y_{j}}$ for any $j<i$. Then $\left\{Z_{i}\right\}_{i=1}^{n}$ form a partition of $Z$. 
Step 2. Let us change the choice variables in the following way:

(i) For every $i=1, \ldots, k-1$ and any $z \in Z_{i}$, given the intermediate effort levels, the Bayes rule defines a one-to-one correspondence between $\left\{\zeta_{y_{j}}(z)\right\}_{j=1}^{k}$ and $\left\{\zeta_{y_{i}}(z), P_{y_{i+1}}(z), \ldots, P_{y_{k}}(z)\right\}$. To see this, note that for any $j=i+1, \ldots, k$,

$$
\zeta_{y_{j}}(z)=M_{j i}(z) \zeta_{y_{i}}(z)
$$

where

$$
M_{j i}(z)=\frac{P_{y_{j}} \operatorname{Pr}\left(y_{i} \mid e_{1}, \ldots, e_{n}\right)}{\left(1-\sum_{\ell=i+1}^{k} P_{y_{\ell}}\right) \operatorname{Pr}\left(y_{j} \mid e_{1}, \ldots, e_{n}\right)},
$$

and $\operatorname{Pr}\left(y_{i} \mid e_{1}, \ldots, e_{n}\right)$ is the probability of outcome $y_{i}$ conditional on the intermediate effort levels $e_{1}, \ldots, e_{n}$. Note that $M_{j i}$ depend only on $P_{y_{j}}$ for $j=i+1, \ldots, k$ and $e_{1}, \ldots, e_{n}$. So for $z \in Z_{i}$ the firm can maximize with respect to $\left\{\zeta_{y_{i}}(z), P_{y_{i+1}}(z), \ldots, P_{y_{k}}(z)\right\}$ instead of $\left\{\zeta_{y_{j}}(z)\right\}_{j=1}^{k}$

So the firm can change the choice variables from $(Z, \zeta)$ to

$$
\left\{Z,\left\{\zeta_{y_{i}}(z), P_{y_{i+1}}(z), \ldots, P_{y_{k}}(z)\right\}_{z \in Z_{i}, i=1, \ldots, k-1},\left\{\zeta_{y_{k}}(z)\right\}_{z \in Z_{k}}\right\}
$$

and add the constraints that $P_{y_{i}}(z) \geq 0$ and $\sum_{i=1}^{k} P_{y_{i}}(z)=1$.

Step 3. Let

$$
\left\{Z^{*},\left\{\zeta_{y_{i}}^{*}(z), P_{y_{i+1}}^{*}(z), \ldots, P_{y_{k}}^{*}(z)\right\}_{z \in Z_{i}, i=1, \ldots, k-1},\left\{\zeta_{y_{k}}^{*}(z)\right\}_{z \in Z_{k}}\right\}
$$

and $\left\{e_{i}^{*}, E_{i}^{*}(z)\right\}_{i=1, \ldots, n}$ be an equilibrium, where $Z^{*}$ can be an infinite set. Then

$$
\left\{Z^{*},\left\{\zeta_{y_{i}}(z), P_{y_{i+1}}^{*}(z), \ldots, P_{y_{k}}^{*}(z)\right\}_{z \in Z_{i}, i=1, \ldots, k-1},\left\{\zeta_{y_{k}}(z)\right\}_{z \in Z_{k}}\right\}
$$

and $\left\{e_{i}^{*}, E_{i}^{*}(z)\right\}_{i=1, \ldots, n}$ will also be an equilibrium if the first-order conditions for the intermediate effort hold, as well as the following conditions:

$$
\begin{gathered}
\sum_{i=1}^{n} e_{i}^{*}+\sum_{j=1}^{k} \operatorname{Pr}\left(y_{j} \mid e_{1}^{*}, \ldots, e_{n}^{*}\right) \int \sum_{i=1}^{n} E_{i}^{*}(z) d \zeta_{y_{j}}(z)= \\
\sum_{i=1}^{n} e_{i}^{*}+\sum_{j=1}^{k} \operatorname{Pr}\left(y_{j} \mid e_{1}^{*}, \ldots, e_{n}^{*}\right) \int \sum_{i=1}^{n} E_{i}^{*}(z) d \zeta_{y_{j}}^{*}(z), \\
\zeta_{y_{j}}(z) \geq 0, \forall z \in Z, j=1, \ldots, k, \\
\int d \zeta_{y_{j}}(z)=1, j=1, \ldots, k .
\end{gathered}
$$

where $\left\{\zeta_{y_{j}}(z), j=i+1, \ldots, k\right\}_{z \in Z_{i}, i=1, \ldots, k-1}$ are functions of the rest of the variables as defined in Step 2 (the first-order conditions for the final-stage effort will hold, because $P_{y_{i}}^{*}$ and $E_{i}^{*}$ are part of an equilibrium). The next step is to show that there exist distributions $\left\{\zeta_{y}\right\}$ with a finite support such that the first-order conditions for the intermediate-stage effort, $(A 1),(A 2)$ and $(A 3)$ hold.

Step 4. Take the first-order conditions for the intermediate-stage effort, $(A 1)$ and $(A 3)$ $(n+k+1$ equations in total: $n$ for the first-order conditions for the intermediate-stage effort, one for $(A 1)$ and $k$ for $(A 3))$ and divide both sides by $\sum_{j=1}^{k} \int d \zeta_{y_{j}}^{*}(z)$. The result will be 
$n+k+1$ equations with respect to the variables $\left\{\zeta_{y_{j}}(z)\right\}_{z \in Z_{j}}, j=1, \ldots, k$, that say that a constant vector on the left-hand side lies in the convex hull of some set, the points of which are indexed by $z \in Z$. Consequently, the left-hand side of this system of equations can be represented as a convex combination of points in this set, where the coefficients of the convex combination are of the form

$$
\frac{1}{\sum_{j=1}^{k} \int d \zeta_{y_{j}}^{*}(z)} \times\left(\left\{\zeta_{y_{j}}(z)\right\}_{z \in Z_{j} \cap Z^{\prime}}\right)
$$

where $Z^{\prime}$ is a finite subset of $Z$. This convex combination defines a probability distribution concentrated on a finite number of points in $Z$. By Caratheodory's theorem, one can choose such a distribution so that it puts positive probability on not more than $n+k+2$ points in $Z$ (since we have $n+k+1$ equations).

Step 5. Now let us prove that, without loss of generality, we can take $|Z| \leq n+k$. Step 3 proves that there exists an optimal disclosure policy that puts positive probability on at most $n+k+2$ signals in $Z$. Let the equilibrium generated by this optimal disclosure policy be

$$
\left\{Z^{*},\left\{\zeta_{y_{i}}^{*}(z), P_{y_{i+1}}^{*}(z), \ldots, P_{y_{k}}^{*}(z)\right\}_{z \in Z_{i}, i=1, \ldots, k-1},\left\{\zeta_{y_{k}}^{*}(z)\right\}_{z \in Z_{k}}\right\}
$$

and $\left\{e_{i}^{*}, E_{i}^{*}(z)\right\}_{i=1, \ldots, n}$. Then $\left\{\zeta_{y_{j}}(z)\right\}_{z \in Z_{j}}, j=1, \ldots, k$, solves

$$
\max _{\zeta} \sum_{i=1}^{n} e_{i}^{*}+\sum_{j=1}^{k} \operatorname{Pr}\left(y_{j} \mid e_{1}^{*}, \ldots, e_{n}^{*}\right) \int \sum_{i=1}^{n} E_{i}^{*}(z) d \zeta_{y_{j}}(z)
$$

subject to $(A 2),(A 3)$ and the first-order conditions for the intermediate effort. This is a linear programming problem, and the canonical form of this problem is

$$
\max _{\alpha} c \alpha \text { s.t. } A \alpha=b, \alpha \geq 0 \text {, }
$$

where $\alpha=\left\{\zeta_{y_{j}}(z)\right\}_{z \in Z_{j}}, j=1, \ldots, k, A$ is a $(n+k) \times(n+k+2)$ matrix (there are $n+k$ constraints, $n$ corresponding to the first-order conditions for the intermediate effort and $k$ to $(A 3)), b \in \mathbb{R}^{n+k}$, and $c \in \mathbb{R}^{n+k+2}$. By the Fundamental Theorem of Linear Programming (see, e.g., Almon, 1967, p. 60), if a solution to this problem exists, then there exists a solution where at most $n+k$ coordinates of $\alpha$ are strictly positive.

B. Justification for the tournament's exogenous reward structure. In the model we have assumed that the reward structure of the tournament is exogenously fixed. In this appendix, we present an extended version of our basic model where the inflexibility of the reward structure of the tournament is derived from the model rather than simply assumed.

Suppose that the workers are ex ante identical but have "types" that govern their productivity. The type of a worker is unknown to all players at the outset of the game but after the hiring, a worker's "type" is revealed and becomes common knowledge for the firm and both workers. Types can be either "high" or "low" and a priori both types are equally likely; i.e., $\operatorname{Pr}($ high $)=1 / 2$.

There are two periods, 1 and 2, and two jobs-Job 1 and Job 2. In period 1, both workers, irrespective of their types, are assigned to Job 1, which is the entry-level job. At the beginning of period 2, a high-type worker may be promoted to job 2. ${ }^{24}$ At the beginning of period 2, after the promotion decision is made public, the firms in the outside labor market compete

\footnotetext{
${ }^{24}$ Even though the low-type workers are not eligible for promotion, as it will be apparent shortly, their presence plays an important role in creating the tournament incentives for the high-type workers.
} 
in wages to raid (or "poach") a worker. However, the market can only observe a worker's job placement but not his type.

The technology is similar to the one described in the basic model except one key difference. In period 1, only a high-type worker can have a success. The probability of success of a hightype worker in both the intermediate and the final stage is equal to the effort he exerts in the respective stage (i.e., the technology assumed in the basic model). In contrast, a low-type worker always fails in both stages.

In period 2, productivity is governed only by the type of the worker (i.e., effort does not play any role). Once a high-type worker completes the two tasks in job 1 , he is more productive in job 2. In particular, a high-type worker produces value $v>1 / 5$ for the firm in job 1 and value $1+v$ in job 2 (as will be made clear later, the assumption $v>1 / 5$ simplifies the subsequent analysis). A high-type worker can also leave the firm to work for another firm in the outside labor market. In the outside labor market, a high-type worker produces 0 in job 1 and 1 in job 2 (so, $v$ can be interpreted as the extent of firm-specific matching). To simplify the subsequent analysis, we assume that a low-type worker produces 0 in all jobs with both the firm and the outside market. The firm can promote only one high-type worker to job 2 , giving rise to a promotion tournament when both workers turn out to be of the high type. A low-type worker is never promoted.

Similar to the spot market contracting assumption in the promotion tournament literature (e.g. Zábojník and Bernhardt, 2001, DeVaro and Waldman, 2007), we assume that the wages following promotion announcement are set through an offer-counteroffer game. Observing the job assignment, the outside labor market makes a wage offer to a worker. The firm observes the offer and decides whether to match the highest offer or let the worker leave for the raider. Consequently, wages are tied to the jobs, and we denote the second-period wage for job $i$ as $w_{i}, i=1,2$.

The payoffs for the workers and the firm are defined as follows. As before, the workers' payoff is their expected reward from the tournament net of the cost of effort (note that the rewards of the tournament are now $w_{1}$ and $w_{2}$ rather than 1 and 0 ). The firm's payoff, $\Pi$, is now the sum of its period 1 and period 2 payoffs. The period 1 payoff, $\pi_{1}$, is the same as the one defined in our basic model. The firm's period 2 expected profit, $\pi_{2}$, is the productivity of the worker in his job net of wages when the worker is retained, and 0 otherwise. So, the payoff from worker $i$ in period 2 is:

$$
\pi_{2}^{i}=\left\{\begin{array}{ll}
1+v-w_{2} & \text { if } i \text { is high-type and promoted to job } 2 \\
v-w_{1} & \text { if } i \text { is high-type and retained in job } 1 \\
-w_{1} & \text { if } i \text { is low-type and retained in job } 1 \\
0 & \text { otherwise }
\end{array} .\right.
$$

Hence, $\pi_{2}=\mathbb{E}\left(\pi_{2}^{A}+\pi_{2}^{B}\right)$ and $\Pi=\pi_{1}+\pi_{2}$.

The strategies of the players are also defined accordingly. The strategy of $F$ will now have two components: (i) the choice of wages $\left(W_{A}, W_{B}\right)$ and a disclosure policy $(Z, \zeta)$ at the beginning of the game, and (ii) the choice of a counteroffer upon observing the market's offer at the beginning of period 2. A worker's strategy now has four components: (i) acceptance or rejection of the firm's offer, (ii) the choice of intermediate-stage effort level, $e_{i}$, given the types, (iii) the choice of final-stage effort level, $E_{i}(z)$, given the types and the realized signal, and (iv) the choice of the period 2 employer upon observing the market offer and the firm's counteroffer. Finally, the outside labor market is assumed to be a non-strategic player that always bids the maximum feasible wage between 0 and 1 subject to the zero-expected-profit constraint given the initial employer's strategy and the observed promotion decision. ${ }^{25}$

\footnotetext{
${ }^{25}$ This restriction is similar in spirit with the "market-Nash" refinement proposed by Waldman (1984). While the key economic effects that we highlight in this article continue to hold even if the outside market is
} 
We keep all other aspects of our basic model unchanged. The following lemma characterizes the reward structure of the promotion tournament.

Lemma 3. In any equilibrium, $w_{1}=0, w_{2}=1$ and there is no turnover.

The argument behind this lemma is as follows. The outside labor market only observes the job assignment and not the type of the worker. Now, a worker being in job 2 necessarily implies that he is a high type, and hence produces a value of 1 with the outside labor market. Because the raiding firms and the initial employer compete in wages to hire the worker, the raiders bid the full value of the worker. The firm finds it optimal to match the offer because by retaining the worker in job 2, the firm makes a profit of $v$ on him.

For a worker in job 1, the outside labor market cannot observe whether the worker is a low type (and produces 0 on all jobs in the outside labor market) or a high type who has lost in the promotion tournament (and can produce an output of value 1 if moved to job 2 in the outside labor market). Because the initial employer observes the type of the worker, this information asymmetry creates an adverse selection problem (see, e.g., Greenwald, 1986). Note that the average productivity of these two types of workers in the outside labor market (when employed in job 2) is $\operatorname{Pr}$ (high-type $\mid$ assigned to Job 1 ) $=1 / 5 .^{26}$ But, recall that with the initial employer, in job 1 , a high-type worker produces $v>1 / 5$ and a low-type worker produces 0 . So, when the outside labor market bids the average productivity of the worker, the firm would always match the offer for a high-type worker, but not for a low-type worker. Consequently, in equilibrium, the market would lower its bid to 0, i.e., the productivity of the low-type worker. The firm will match this offer and make a profit of $v$ by retaining the high-type worker. This effect is similar to the "winner's curse" problem in common-value auctions (McAfee and McMillan, 1987).

Lemma 3 suggests that in this model, the reward structure of the promotion tournament is identical to the one assumed in our basic model. So, our basic model can be conceived as a reduced form version of the more elaborate model described above and representing the case where both workers are revealed to be of the high type.

\section{REFERENCES}

[1] Almon, C. (1967), Matrix Methods in Economics, Cambridge, MA: Addison-Wesley.

[2] Aoyagi, M. (2010) "Information Feedback in a Dynamic Tournament," forthcoming, Games and Economic Behavior.

[3] Beer, M. (1987) "Performance Appraisals," in Lorsch, J. (ed.), Handbook of Organizational Behavior, Englewood Cliffs, NJ: Printice Hall, Inc., pp. 286-301.

[4] __. (1987) "Performance Appraisal," mimeo, Harvard Business School.

[5] Carmichael, L. (1983a) "Firm-Specific Human Capital and Promotion Ladders," The Bell Journal of Economics, 14(1), pp. 251-258.

[6] __ (1983b) "The Agent-Agents Problem: Payment by Relative Output," Journal of Labor Economics, $1(1)$, pp. 50-65.

[7] DeVaro, J., and M. Waldman (2007) "The Signaling Role of Promotions: Further Theory and Empirical Evidence," Cornell University ILR School working paper.

[8] Dubey, P., and J. Geanakoplos (2010) "Grading Exams: 100,99,..,1 or A, B, C? Incentives in Games of Status," forthcoming, Games and Economic Behavior.

[9] Ederer, F. (2010) "Feedback and Motivation in Dynamic Tournaments," forthcoming, Journal of Economics and Management Strategy.

[10] Fuchs, W. (2007) "Contracting with Repeated Moral Hazard and Private Evaluations," American Economic Review, 97, pp. 1432-1448.

fully strategic, this restiction simplifies the analysis by ruling out certain intuitively unappealing outcomes of the offer-counteroffer game.

${ }^{26}$ This probability can be obtained by using Bayes rule under the assumption that both workers stay in Job 1 when both are of low-type. 
[11] Fudenberg, D., R. Gilbert, J. Stiglitz and J. Tirole (1983) "Preemption, Leapfrogging, and Competition in Patent Races," European Economic Review, 22, pp. 3-31.

[12] Gershkov, A., and M. Perry (2009) "Tournaments with Midterm Reviews," Games and Economic Behavior, 66, pp. 162-190.

[13] Gibbs, M. (1991) "An Economic Approach to Process in Pay and Performance Appraisals," mimeo, University of Chicago, Graduate School of Business.

[14] Green, E., and N. Stokey (1983) "A Comparison of Tournaments and Contracts," Journal of Political Economy, 91, pp. 349-364.

[15] Greenwald, B. (1986) "Adverse Selection in the Labor Market," Review of Economic Studies, 53, pp. 325-347.

[16] Hamner, W. (1975) "How to Ruin Motivation with Pay," Compensation Review, 7, pp. 17-27.

[17] Hansen, S. (2009) "What They Don't Know Can't Hurt You: The Benefits of Limited Feedback in Organizations," mimeo.

[18] Harris, C., and J. Vickers (1987) "Racing with Uncertainty," Review of Economic Studies, 54(1), pp. 1-21.

[19] Lawler, E. (1972) "Secrecy and the Need to Know," in Tosi, House and Dunnette (eds.), Managerial Motivation and Compensation, MSU Business Studies.

[20] Lazear, E., and S. Rosen (1981) "Rank Order Tournaments as Optimal Labor Contracts," Journal of Political Economy, 89, pp. 841-864.

[21] Lizzeri, A., M. Meyer and N. Persico (2002) "The Incentive Effects of Interim Performance Evaluations," CARESS working paper \#02-09.

[22] Loveman, W. and J. O'Connell "HCL America." Harvard Business Review, Case \# 9-396-030 (1996).

[23] McAfee, P. R. and J. McMillan (1987) "Auctions and Bidding," Journal of Economic Literature, 25, 699-738.

[24] MacLeod, B. (2003) "Optimal Contracting with Subjective Evaluation," American Economic Review, 93(1), pp: 216-240.

[25] Malcomson, J. (1984) "Work Incentives, Hierarchy, and Internal Labor Markets," Journal of Political Economy, 92(3), pp. 486-507.

[26] _ _ (1986) "Rank-Order Contracts for a Principal with Many Agents," Review of Economic Studies, 53(5), pp. 807-817.

[27] Meyer, M. A. (1991) "Learning from Coarse Information: Biased Contests and Career Profiles," Review of Economic Studies, 58, pp. 15-41.

[28] _ _ (1992) "Biased Contests and Moral Hazard: Implications for Career Profiles," Annales d'Economie et de Statistique, 25/26, pp. 165-187.

[29] Murphy, K. (1990) "Merck and Co., Inc. (A), (B), and (C)," Harvard Business School cases \# 9-491-005, 006 , and 007.

[30] __ (1992) "Performance Measurement and Appraisal: Motivating Managers to Identify and Reward Performance," in William J. Bruns, Jr. (ed.), Performance Measurement, Evaluation, and Incentives, Boston: Harvard Business School Press.

[31] Nalebuff, B., and J. Stiglitz (1983) "Prizes and Incentives: Towards a General Theory of Compensation and Competition," The Bell Journal of Economics, 14, pp. 21-43.

[32] Peiperl, M. (2001) "Getting 3600 Feedback Right," Harvard Business Review, pp. 142-147.

[33] Rosen, S. (1986) "Prizes and Incentives in Elimination Tournaments," American Economic Review, 76(4), pp. 701-715.

[34] Waldman, M. (1984) "Job Assignments, Signalling, and Efficiency Job Assignments, Signalling, and Efficiency," The RAND Journal of Economics, 15(2), pp. 255-267.

[35] __ _ (1990) "Up-or-Out Contracts: A Signaling Perspective," Journal of Labor Economics, 8(2), pp. $230-250$

[36] Yildirim, H. (2005) "Contests with Multiple Rounds," Games and Economic Behavior, 51(1), pp. 213-227.

[37] Zábojník, J. and D. Bernhardt (2001) "Corporate Tournaments, Human Capital Acquisition, and the Firm Size-Wage Relation," The Review of Economic Studies, 68(3), pp. 693-716. 